\title{
Thermodynamic formula for the cumulant generating function of time-averaged current
}

\author{
Takahiro Nemoto and Shin-ichi Sasa \\ Department of pure and applied Sciences, The University of Tokyo, Tokyo, 153-8902, Japan
}

(Dated: October 23, 2018)

\begin{abstract}
The cumulant generating function of time-averaged current is studied from an operational viewpoint. Specifically, for interacting Brownian particles under non-equilibrium conditions, we show that the first derivative of the cumulant generating function is equal to the expectation value of the current in a modified system with an extra force added, where the modified system is characterized by a variational principle. The formula reminds us of Einstein's fluctuation theory in equilibrium statistical mechanics. Furthermore, since the formula leads to the fluctuation-dissipation relation when the linear response regime is focused on, it is regarded as an extension of the linear response theory to that valid beyond the linear response regime. The formula is also related to previously known theories such as the Donsker-Varadhan theory, the additivity principle, and the least dissipation principle, but it is not derived from them. Examples of its application are presented for a driven Brownian particle on a ring subject to a periodic potential.
\end{abstract}

PACS numbers: 05.40.-a, 05.70.Ln, 02.50.Ey

\section{INTRODUCTION}

Entropy $S$ as a function of extensive variables characterizes macroscopic properties of materials in a unified manner [1]. Its functional form for a given material is completely determined by thermodynamic measurements of the heat capacity, the compressibility and the other relevant susceptibilities. Let $X$ be an unconstrained variable of a composite isolated system. We denote the $X$ dependence of $S$ by $S(X)$. Then, the equilibrium value of $X$ is determined by

$$
X_{*}=\underset{X}{\operatorname{argmax}}[S(X)],
$$

which is an example of the variational principle of thermodynamics. Furthermore, according to Einstein's fluctuation theory [1], the probability density of $X$ is expressed as

$$
\operatorname{Prob}(X) \simeq e^{\left(S(X)-S\left(X_{*}\right)\right) / k_{\mathrm{B}}},
$$

where $k_{\mathrm{B}}$ is the Boltzmann constant, and $\simeq$ represents the asymptotic equivalence of the exponential factor in the thermodynamic limit. More precisely, let $N$ be the number of the degrees of freedom. Then, the scaled entropy $s(x)=\lim _{N \rightarrow \infty} S(X) / N$ with $x=X / N$ fixed satisfies

$$
\frac{1}{k_{\mathrm{B}}}\left(s(x)-s\left(x_{*}\right)\right)=\lim _{N \rightarrow \infty} \frac{1}{N} \log \operatorname{Prob}(x) .
$$

In the probability theory, the right-hand side provides a large deviation function (up to the minus sign) [2]. That is, the scaled entropy $s(x)$ is equivalent to the large deviation function.

Now, suppose that the scaled entropy $s(x)$ is defined by (3) with the principle of the equal weight for microscopic states. Then, the variational principle (1) can be derived from the definition. A surprise in this approach is that the entropy thus defined characterizes thermodynamic properties completely through the fundamental relation of thermodynamics. After establishing the connection to thermodynamics, one can obtain the scaled entropy by thermodynamic measurements. In other words, the large deviation function, which characterizes macroscopic fluctuations, is obtained without measuring fluctuations. Such formulas are not restricted to the case of fluctuations in isolated systems. As demonstrated for a simple model in Appendix A, several formulas are derived. One example is that a scaled cumulant generating function of a thermodynamic variable, which is given by the Legendre transform of the large deviation function, is related to a free energy density in thermodynamics. From this relation, it is found that the derivative of the scaled cumulant generating function with respect to its argument is equal to the expectation value of the thermodynamic variable in a modified system with an extra external force added. See (A9) for such an example. The relations between large deviation functions and thermodynamic functions are thought to be well-known, and the relations lead to fluctuation-response relations, one of which, connects the susceptibility and the intensity of fluctuations of magnetization [1].

The main difference of non-equilibrium systems from equilibrium systems is the existence of a current. In particular, the expectation value of the current in a linear response regime is connected with the intensity of fluctuations of time-averaged current in equilibrium [3, 4]. This is one of fluctuation-dissipation relations, which might correspond to fluctuation-response relations in equilibrium statistical mechanics. Since the time-averaged current appears to be important at least for small fluctuations in the linear response regime, it is natural to consider the large deviation function of time-averaged current. Then, from the analogy with thermodynamics, one may expect that there is a formula that connects the large deviation function with a macroscopic quantity and that 
the fluctuation-dissipation relations are derived from the formula when the linear response regime is focused on. Indeed, Onsager and Onsager-Machlup attempted to express the large deviation function of time-averaged current in terms of dissipation functions [5-7], as pointed out by Oono [8]. This is successful for small fluctuations in the linear response regime, but the expression cannot be applied to general cases with large fluctuations.

In the last two decades, the understanding of the large deviation function of time-averaged current has been substantially developed. One remarkable result is the discovery of the fluctuation theorem which represents a simple and beautiful symmetry property of the large deviation function of time-averaged entropy production [9]. The symmetry property was understood from the microscopic reversibility of the system and it was generalized to identities for any quantities $[10$ 14]. This progress enables us to revisit a linear response theory and to re-derive known formulas including a non-linear response formula 15] and an expression of steady state distribution [16, 17]. See Ref. [18] as a review of these re-derivations. In addition to the re-derivation of the known formulas, the symmetry property plays an essential role in deriving an extension of the Clausius relation [19 21] on the basis of a neat expression of steady state distribution [22]. As another direction of the progress, the large deviation function of time-averaged current was explicitly calculated for lattice models [23, 24]. The results evolved into a variational form, which was called an additivity principle 2528$]$. When the system behavior is assumed to be described by fluctuating hydrodynamics, the condition for the validity of the additivity principle can be derived from the analysis of the path-integral expression [29 33]. Note that the additivity principle is less universal than the fluctuation theorem, but still characterizes a universal aspect of the large deviation functions of time-averaged current in a wide range class of systems. See Ref. [34] for a recent related study.

Based on these developments, we consider a possibility that the large deviation function of time-averaged current is expressed by a time-averaged quantity as if the framework corresponds to Einstein's fluctuation theory. This formula, if it exists, is regarded as an extension of Onsager's theory to that valid even for large fluctuations. Although the motivation seems natural, the problem would not be solved easily. Nevertheless, since a new operational variational principle associated with the large deviation function of time-averaged current has been found recently [35], it is a good opportunity to study the problem.

In this paper, specifically, we study interacting Brownian particles under non-equilibrium conditions. The motion of the particles are assumed to be described by a set of Langevin equations. We then consider the large deviation function of time-averaged velocity of the particles. The main results in this paper are formulas (26) and (28). The formula (26) indicates that the scaled cumulant generating function, which is given by the Legendre transform of the large deviation function, is determined from a variational function in a modified system with an inhomogeneous external force added. Essentially the same formula was reported in Ref. [35]. The formula (28) claims that the derivative of the scaled cumulant generating function is equal to the time-averaged velocity in the modified system, which corresponds to (A9) in equilibrium statistical mechanics. The formula (28) also turns out to be an extension of the fluctuation-dissipation relation. Since these new formulas take simple forms, we expect that our results advance the understanding of large deviation function of time-averaged quantity.

This paper is organized as follows. In section [1] we introduce a model and define quantities we study in this paper. In section III, we first summarize main results of the paper and after that we derive them. In section IV, we address three remarks on the variational function that appears in our main results. Concretely, we re-express the variational function in terms of entropy production rates, mention a similarity with the Donsker-Varadhan formula, and explain a connection to the principle of the least dissipation of energy. In section $\mathrm{V}$, we present a few applications of our formula by restricting our study to a single Brownian particle on a ring. First, on the basis of our formula, we re-derive exact formulas of the diffusion constant and the mobility in a compact manner. Second, starting from our formula, we show a variational expression associated with an additivity principle. Third, we propose a method for experimentally obtaining the scaled cumulant generating function. The final section is devoted to concluding remarks. Some technical details and related subjects are discussed in Appendices. In the argument below, the Boltzmann constant $k_{\mathrm{B}}$ is set to unity.

\section{MODEL}

We consider $N$ colloidal particles immersed in a solvent at temperature $T$. We describe the system by a set of positions of the particles in a $d$-dimensional region $\Omega$. For mathematical simplicity, we impose periodic boundary conditions. As a typical example, one may consider a three-dimensional region, where one direction is confined by walls and no walls in other directions. Even such a case is included in our model by setting up a periodic potential whose height is extremely large at the walls. Another example of the region $\Omega$ is a one-dimensional circuit which can be generated by an optical potential.

We denote the positions of all the particles by $\boldsymbol{x} \equiv$ $\left(x_{i}\right)_{i=1}^{N d}$, where $x_{(k-1) d+l}$ represents the $l$-th component of the position of the $k$-th particle. In this paper, boldface fonts (e.g. $\boldsymbol{x}$ ) represent $N d$-dimensional vectors. We also express the position of the $k$-th particle by the $d$ dimensional vector $\vec{r}_{k}=\left(x_{(k-1) d+1}, x_{(k-1) d+2}, \ldots, x_{k d}\right)$.

The force acting on the particles consists of three types of force, (i) an external force, (ii) an interaction force exerted from other particles, and (iii) a force from solvent 
molecules. In (i), the external force is further divided into a homogeneous part $\boldsymbol{f}=\left(f_{i}\right)_{i=1}^{N d}$ and a periodic part determined by a potential $\sum_{k=1}^{N} U_{k}\left(\vec{r}_{k}\right)$. Since the existence of $\boldsymbol{f}$ makes the system out of equilibrium, we call $\boldsymbol{f}$ a driving force. In (ii), the interaction force is determined by a two-body interaction potential $U_{k, l}\left(\vec{r}_{k}-\vec{r}_{l}\right)$ between the $k$-th particle and the $l$-th particle under the assumption that three- and higher-body interactions among particles are not taken into account. The total potential $U(\boldsymbol{x})$ is written as

$$
U(\boldsymbol{x})=\sum_{k=1}^{N} U_{k}\left(\vec{r}_{k}\right)+\frac{1}{2} \sum_{k=1}^{N} \sum_{l \neq k} U_{k, l}\left(\vec{r}_{k}-\vec{r}_{l}\right) .
$$

The total force $\boldsymbol{F}(\boldsymbol{x})$ that originates from (i) and (ii) is expressed as

$$
\boldsymbol{F}(\boldsymbol{x})=\boldsymbol{f}-\frac{\partial}{\partial \boldsymbol{x}} U(\boldsymbol{x}) .
$$

In (iii), the force from solvent molecules is divided into two parts, a friction force and a fluctuating force, where we assume that hydrodynamic interactions are ignored. The friction force is proportional to the particle velocity $\dot{x}_{i}=d x_{i} / d t$ so that it can be written in the form of $-\dot{x}_{i} \gamma_{i}$ with friction constants $\left(\gamma_{i}\right)_{i=1}^{N d}$. We assume that the fluctuating force is described as Gaussian white noise satisfying the fluctuation-dissipation relation of the second kind. That is, it is expressed by $\sqrt{2 T \gamma_{i}} \xi_{i}(t)$, where $\xi_{i}(t)$ satisfies

$$
\begin{gathered}
\left\langle\xi_{i}(t)\right\rangle=0, \\
\left\langle\xi_{i}(t) \xi_{j}(s)\right\rangle=\delta_{i, j} \delta(t-s) .
\end{gathered}
$$

From these arguments, we obtain the equations of motion for colloidal particles. By neglecting inertial effects, these are written as

$$
\dot{x}_{i}=\frac{1}{\gamma_{i}} F_{i}(\boldsymbol{x})+\sqrt{\frac{2 T}{\gamma_{i}}} \xi_{i}
$$

where $i=1,2, \cdots, N d$. The equations are called Langevin equations [36] and have been studied in many situations including Brownian motors [37], polymer dynamics [38] and dynamics of mesoscale objects in solids. See Introduction of Refs. [39, 40] and Chap. 11 of Ref. [45]. It should be noted that some recent discoveries of non-equilibrium relations have been tested in experiments corresponding to (8) with $N=d=1$ [41 43].

We assume that the particles obey a probability density $P_{0}(\boldsymbol{x})$ at $t=0$. After that $(t>0)$, the particles move according to (8). We denote by $\langle f\rangle$ the expectation value of $f$ with respect to the initial distribution $P_{0}(\boldsymbol{x})$ and history of $\xi_{i}(t)$. We define the probability density of $\boldsymbol{x}(t)=\boldsymbol{x}$ by $P^{\boldsymbol{F}}(\boldsymbol{x}, t) \equiv\langle\delta(\boldsymbol{x}(t)-\boldsymbol{x})\rangle$. Here, the superscript $\boldsymbol{F}$ of $P^{\boldsymbol{F}}(\boldsymbol{x}, t)$ represents $\boldsymbol{F}(\boldsymbol{x})$ in (8). We use this superscript throughout the paper, because the force dependence plays an important role in our formulation. The evolution equation for $P^{\boldsymbol{F}}(\boldsymbol{x}, t)$ is derived as

$$
\frac{\partial P^{\boldsymbol{F}}(\boldsymbol{x}, t)}{\partial t}=-\sum_{i=1}^{N d} \frac{\partial j_{i}^{\boldsymbol{F}}(\boldsymbol{x}, t)}{\partial x_{i}}
$$

with a probability current

$$
j_{i}^{\boldsymbol{F}}(\boldsymbol{x}, t)=\frac{P^{\boldsymbol{F}}(\boldsymbol{x}, t)}{\gamma_{i}} F_{i}(\boldsymbol{x})-\frac{T}{\gamma_{i}} \frac{\partial P^{\boldsymbol{F}}(\boldsymbol{x}, t)}{\partial x_{i}} .
$$

The equation is called the Fokker-Planck equation [36].

The stationary distribution and current, which are denoted by $P_{\mathrm{st}}^{\boldsymbol{F}}(\boldsymbol{x})$ and $J_{\mathrm{st}, i}^{\boldsymbol{F}}(\boldsymbol{x})$, respectively, are determined by

$$
\begin{aligned}
J_{\mathrm{st}, i}^{\boldsymbol{F}}(\boldsymbol{x})= & \frac{P_{\mathrm{st}}^{\boldsymbol{F}}(\boldsymbol{x})}{\gamma_{i}} F_{i}(\boldsymbol{x})-\frac{T}{\gamma_{i}} \frac{\partial P_{\mathrm{st}}^{\boldsymbol{F}}(\boldsymbol{x})}{\partial x_{i}}, \\
& \sum_{j=1}^{N d} \frac{\partial}{\partial x_{j}} J_{\mathrm{st}, j}^{\boldsymbol{F}}(\boldsymbol{x})=0 .
\end{aligned}
$$

The expectation value of a quantity $A\left[(\boldsymbol{x}(s))_{s=0}^{t}\right]$ in the steady state is denoted by $\langle A\rangle_{\text {st }}^{\boldsymbol{F}}$. Mathematically, the expectation value is calculated by replacing initial distribution $P_{0}(\boldsymbol{x})$ by $P_{\text {st }}^{\boldsymbol{F}}(\boldsymbol{x})$.

In this paper, we particularly focus on statistical properties of time-averaged velocities

$$
\boldsymbol{V}(\tau) \equiv \frac{1}{\tau} \int_{0}^{\tau} d t \dot{\boldsymbol{x}}(t)
$$

The scaled cumulant generating function $G^{\boldsymbol{F}}(\boldsymbol{h})$ of the time-averaged velocities is defined by

$$
G^{\boldsymbol{F}}(\boldsymbol{h}) \equiv \lim _{\tau \rightarrow \infty} \frac{1}{\tau} \log \left\langle e^{\tau \sum_{i=1}^{N d} h_{i} V_{i}(\tau)}\right\rangle,
$$

where $\boldsymbol{h}=\left(h_{i}\right)_{i=1}^{N d}$. In the expansion form

$$
G^{\boldsymbol{F}}(\boldsymbol{h})=\sum_{k_{1}=0}^{\infty} \sum_{k_{2}=0}^{\infty} \ldots \sum_{k_{N d}=0}^{\infty} C_{k_{1}, k_{2}, \ldots, k_{N d}}^{\boldsymbol{F}} \frac{h_{1}^{k_{1}} h_{2}^{k_{2}} \ldots h_{N d}^{k_{N d}}}{k_{1} ! k_{2} ! \ldots k_{N d} !}
$$

the coefficient $C_{k_{1}, k_{2}, \ldots, k_{N d}}^{\boldsymbol{F}}$ is related to the cumulant $\left\langle V_{1}^{k_{1}}(\tau) V_{2}^{k_{2}}(\tau) \ldots V_{N d}^{k_{N d}}(\tau)\right\rangle_{\mathrm{c}}$ [36]. Concretely, it can be shown that

$$
C_{k_{1}, k_{2}, \ldots, k_{N d}}^{\boldsymbol{F}}=\lim _{\tau \rightarrow \infty}\left\langle V_{1}^{k_{1}}(\tau) V_{2}^{k_{2}}(\tau) \ldots V_{N d}^{k_{N d}}(\tau)\right\rangle_{\mathrm{c}} \tau^{k-1} .
$$

Here, by substituting (8) into (13) and taking the expectation value, we obtain

$$
\left\langle V_{i}(\tau)\right\rangle=\int_{\Omega^{N}} d \boldsymbol{x} \frac{1}{\gamma_{i}} F_{i}(\boldsymbol{x})\left(\frac{1}{\tau} \int_{0}^{\tau} d t P^{\boldsymbol{F}}(\boldsymbol{x}, t)\right) .
$$

This leads to

$$
\left\langle V_{i}\right\rangle_{\mathrm{st}}^{\boldsymbol{F}}=\int_{\Omega^{N}} d \boldsymbol{x} \frac{1}{\gamma_{i}} F_{i}(\boldsymbol{x}) P_{\mathrm{st}}^{\boldsymbol{F}}(\boldsymbol{x})
$$


Furthermore, by substituting (11) into (18) and by eliminating boundary terms due to periodic boundary conditions, we obtain

$$
\left\langle V_{i}\right\rangle_{\mathrm{st}}^{\boldsymbol{F}}=\int_{\Omega^{N}} d \boldsymbol{x} J_{\mathrm{st}, i}^{\boldsymbol{F}}(\boldsymbol{x}) .
$$

\section{MAIN RESULTS}

The main purpose of this paper is to find an expression of $G^{\boldsymbol{F}}(\boldsymbol{h})$ which shares a common structure in equilibrium statistical mechanics. As reviewed in Appendix A. the cumulant generating function of a thermodynamic variable is related to a free energy function and this relation leads to the important result that the first derivative of the cumulant generating function is equal to the expectation value of the thermodynamic variable in a modified system. See (A9) for such an example. We want to have a similar expression for $G^{\boldsymbol{F}}(\boldsymbol{h})$.

Since the relation containing (A9) leads to the fluctuation-response relation for thermodynamic variables, we expect that there might be a formula similar to (A9), which leads to fluctuation-dissipation relations for currents. In order to investigate this possibility, we focus on the linear response regime. First, by noting $\left.\left(\partial G^{\boldsymbol{F}}(\boldsymbol{h}) / \partial h_{i}\right)\right|_{\boldsymbol{h}=0}=\left\langle V_{i}\right\rangle_{\mathrm{st}}$, we have

$$
\frac{\partial G^{\boldsymbol{F}}(\boldsymbol{h})}{\partial h_{i}}=\left\langle V_{i}\right\rangle_{\mathrm{st}}^{\boldsymbol{F}}+\left.\sum_{j=1}^{N d} h_{j}\left(\frac{\partial^{2} G^{\boldsymbol{F}}(\boldsymbol{h})}{\partial h_{i} \partial h_{j}}\right)\right|_{\boldsymbol{h}=0}+\mathcal{O}\left(\boldsymbol{h}^{2}\right) .
$$

Here, the fluctuation-dissipation relation is written as

$$
\left\langle V_{i}\right\rangle_{\mathrm{st}}^{\boldsymbol{F}}=\left.\frac{1}{2 T} \sum_{j=1}^{N d} f_{j}\left(\frac{\partial^{2} G^{\boldsymbol{F}}(\boldsymbol{h})}{\partial h_{i} \partial h_{j}}\right)\right|_{\boldsymbol{h}=0, \boldsymbol{f}=0}+\mathcal{O}\left(\boldsymbol{f}^{2}\right) .
$$

See Refs. [44, 45] for a derivation. See also Refs. [14, 18] for a recent understanding of the derivation on the basis of the fluctuation theorem. The substitution of (21) into the first term of the right-hand side of (20) and the evaluation of the second term at $\boldsymbol{f}=0$ yield

$$
\begin{aligned}
\frac{\partial G^{\boldsymbol{F}}(\boldsymbol{h})}{\partial h_{i}}= & \left.\frac{1}{2 T} \sum_{j=1}^{N d}\left(f_{j}+2 T h_{j}\right)\left(\frac{\partial^{2} G^{\boldsymbol{F}}(\boldsymbol{h})}{\partial h_{i} \partial h_{j}}\right)\right|_{\boldsymbol{h}=0, \boldsymbol{f}=0} \\
& +\mathcal{O}\left(\boldsymbol{f}^{2}, \boldsymbol{h}^{2}, \boldsymbol{f} \boldsymbol{h}\right) .
\end{aligned}
$$

By using (21) again in the right-hand side of (22), we obtain

$$
\frac{\partial G^{\boldsymbol{F}}(\boldsymbol{h})}{\partial h_{i}}=\left\langle V_{i}\right\rangle_{\mathrm{st}}^{\boldsymbol{F}+2 T \boldsymbol{h}}+\mathcal{O}\left(\boldsymbol{h}^{2}, \boldsymbol{f}^{2}, \boldsymbol{f} \boldsymbol{h}\right) .
$$

This implies that when $\boldsymbol{f}$ and $\boldsymbol{h}$ are small, the first derivative of $G^{\boldsymbol{F}}(\boldsymbol{h})$ is equal to the expectation value of the time-averaged velocity in a modified system with an extra force $2 T \boldsymbol{h}$ added. Since (21) is derived from (20) and
(23), the expression (23) is equivalent to the fluctuationdissipation relation (21).

Now, we seek for an extension of (23) to that valid for any $\boldsymbol{h}$ and $\boldsymbol{f}$. Here, one may recall the fluctuation theorem as an extension of the fluctuation-dissipation relation, which claims

$$
G^{\boldsymbol{F}}(\boldsymbol{h})=G^{\boldsymbol{F}}(-\boldsymbol{h}-\boldsymbol{f} / T)
$$

in the system under consideration [14]. Although (21) is derived from (24), a clear extension of (23) is not obtained as far as we attempted. Putting aside the fluctuation theorem, we consider the problem from a different direction. The basic idea is to consider a modified system with a spatially inhomogeneous extra force $\boldsymbol{w}(\boldsymbol{x})$ added. Precisely writing, the modified system is described by the Langevin equation (8) with the replacement of $\boldsymbol{F}(\boldsymbol{x})$ by $\boldsymbol{F}(\boldsymbol{x})+\boldsymbol{w}(\boldsymbol{x})$. We then define a functional $\Phi_{\boldsymbol{h}}^{\boldsymbol{F}}(\boldsymbol{w})$ by

$$
\Phi_{\boldsymbol{h}}^{\boldsymbol{F}}(\boldsymbol{w}) \equiv \sum_{i=1}^{N d}\left[h_{i}\left\langle V_{i}\right\rangle_{\mathrm{st}}^{\boldsymbol{F}+\boldsymbol{w}}-\frac{1}{4 T \gamma_{i}}\left\langle w_{i}^{2}\right\rangle_{\mathrm{st}}^{\boldsymbol{F}+\boldsymbol{w}}\right] .
$$

The first result of this paper is a variational expression

$$
G^{\boldsymbol{F}}(\boldsymbol{h})=\max _{\boldsymbol{w}} \Phi_{\boldsymbol{h}}^{\boldsymbol{F}}(\boldsymbol{w}) .
$$

Let $\boldsymbol{w}_{\boldsymbol{h}}^{\boldsymbol{F} \text {,opt }}$ be the optimal $\boldsymbol{w}$ that maximizes (25) for given $\boldsymbol{h}$ and $\boldsymbol{F}$. That is,

$$
\boldsymbol{w}_{\boldsymbol{h}}^{\boldsymbol{F}, \mathrm{opt}}=\underset{\boldsymbol{w}}{\operatorname{argmax}}\left[\Phi_{\boldsymbol{h}}^{\boldsymbol{F}}(\boldsymbol{w})\right] .
$$

The second result is a formula

$$
\frac{\partial G^{\boldsymbol{F}}(\boldsymbol{h})}{\partial h_{i}}=\left\langle V_{i}\right\rangle_{\mathrm{st}}^{\boldsymbol{F}+\boldsymbol{w}_{h}^{F, \mathrm{opt}}} .
$$

This is thought to be the generalization of (23). Indeed, there exists a positive function $\psi_{\boldsymbol{h}}(\boldsymbol{x})$ such that

$$
\boldsymbol{w}_{\boldsymbol{h}}^{\boldsymbol{F}, \mathrm{opt}}(\boldsymbol{x})=2 T \boldsymbol{h}+2 T \frac{\partial}{\partial \boldsymbol{x}} \log \psi_{\boldsymbol{h}}(\boldsymbol{x})
$$

with a condition

$$
\lim _{\boldsymbol{h} \rightarrow 0} \frac{\partial}{\partial x_{i}} \log \psi_{\boldsymbol{h}}(\boldsymbol{x})=0 .
$$

Before deriving the main results, we confirm that the fluctuation-dissipation relation (21) is obtained from our result (28) with (29). We start with

$$
\frac{\partial G^{\boldsymbol{F}}(\boldsymbol{h})}{\partial h_{i}}=\left\langle V_{i}\right\rangle_{\mathrm{st}}^{\boldsymbol{f}-\frac{\partial U}{\partial \boldsymbol{x}}+2 T \boldsymbol{h}+2 T \frac{\partial \log \psi_{\boldsymbol{h}}}{\partial \boldsymbol{x}}} .
$$

From a basic property that there is no persistent current in systems without a driving force, we have an identity

$$
\left\langle V_{i}\right\rangle_{\mathrm{st}}^{-\frac{\partial U}{\partial \boldsymbol{x}}+2 T \frac{\partial \log \psi_{\boldsymbol{h}}}{\partial \boldsymbol{x}}}=0 .
$$


This leads to

$$
\begin{aligned}
& \left\langle V_{i}\right\rangle_{\mathrm{st}}^{\boldsymbol{f}+2 T \boldsymbol{h}-\frac{\partial U}{\partial \boldsymbol{x}}+2 T \frac{\partial \log \psi_{\boldsymbol{h}}}{\partial \boldsymbol{x}}} \\
& =\sum_{j=1}^{N d} \lim _{\boldsymbol{f}^{\prime} \rightarrow 0}\left[\frac{\partial\left\langle V_{i}\right\rangle_{\mathrm{st}}^{\boldsymbol{f}^{\prime}-\frac{\partial U}{\partial \boldsymbol{x}}+2 T \frac{\partial \log \psi_{\boldsymbol{h}}}{\partial \boldsymbol{x}}}}{\partial f_{j}^{\prime}}\right]\left(f_{j}+2 T h_{j}\right) \\
& \quad+\mathcal{O}\left((\boldsymbol{f}+2 T \boldsymbol{h})^{2}\right) .
\end{aligned}
$$

By using (30), we further rewrite the right-hand side of (33) as

$$
\sum_{j=1}^{N d} \lim _{\boldsymbol{f}^{\prime} \rightarrow 0}\left[\frac{\partial\left\langle V_{i}\right\rangle_{\mathrm{st}}^{\boldsymbol{f}^{\prime}-\frac{\partial U}{\partial \boldsymbol{x}}}}{\partial f_{j}^{\prime}}\right]\left(f_{j}+2 T h_{j}\right)+\mathcal{O}\left(\boldsymbol{f}^{2}, \boldsymbol{h}^{2}, \boldsymbol{f} \boldsymbol{h}\right) .
$$

Thus, we arrive at

$$
\begin{aligned}
\frac{\partial G^{\boldsymbol{F}}(\boldsymbol{h})}{\partial h_{i}}= & \sum_{j=1}^{N d} \lim _{\boldsymbol{f}^{\prime} \rightarrow 0}\left[\frac{\partial\left\langle V_{i}\right\rangle_{\mathrm{st}}^{\boldsymbol{f}^{\prime}-\frac{\partial U}{\partial \boldsymbol{x}}}}{\partial f_{j}}\right]\left(f_{j}+2 T h_{j}\right) \\
& +\mathcal{O}\left(\boldsymbol{f}^{2}, \boldsymbol{h}^{2}, \boldsymbol{f} \boldsymbol{h}\right) .
\end{aligned}
$$

By differentiating (35) with respect to $h_{j}$ and setting $\boldsymbol{f}=0$ and $\boldsymbol{h}=0$, we obtain

$$
\left.\frac{\partial^{2} G^{\boldsymbol{F}}(\boldsymbol{h})}{\partial h_{i} \partial h_{j}}\right|_{\boldsymbol{f}=0, \boldsymbol{h}=0}=2 T \lim _{\boldsymbol{f}^{\prime} \rightarrow 0}\left[\frac{\partial\left\langle V_{i}\right\rangle_{\mathrm{st}}^{\boldsymbol{f}^{\prime}-\frac{\partial U}{\partial \boldsymbol{x}}}}{\partial f_{j}}\right] .
$$

This is equivalent to (21).

\section{A. Derivation}

The set of displacements of particles during the time interval $[0, t]$ is written as

$$
\boldsymbol{X}(t)=\int_{0}^{t} d t^{\prime} \dot{\boldsymbol{x}}\left(t^{\prime}\right)
$$

where it should be noted that

$$
\boldsymbol{X}(t)=\boldsymbol{V}(t) t .
$$

By differentiating (37) with respect to $t$, we obtain

$$
\frac{d X_{i}}{d t}=\frac{1}{\gamma_{i}} F_{i}(\boldsymbol{x})+\sqrt{\frac{2 T}{\gamma_{i}}} \xi_{i}
$$

We denote the joint probability density of $\boldsymbol{x}(t)$ and $\boldsymbol{X}(t)$ by $p(\boldsymbol{x}, \boldsymbol{X}, t)$. The idea of considering such a joint probability density of $\boldsymbol{x}$ and $\boldsymbol{X}$ (or accumulated work, heat) was employed in Refs. [48 50]. From the Langevin equations (8) and (39), we derive the Fokker-Planck equation

$$
\frac{\partial p}{\partial t}=\mathcal{L}_{\mathrm{FP}}^{(\boldsymbol{x}, \boldsymbol{X})} \cdot p,
$$

where the Fokker-Planck operator $\mathcal{L}_{\mathrm{FP}}^{(\boldsymbol{x}, \boldsymbol{X})}$ is defined by

$$
\begin{aligned}
& \mathcal{L}_{\mathrm{FP}}^{(\boldsymbol{x}, \boldsymbol{X})} \cdot \varphi \\
& =\sum_{i=1}^{N d}\left[-\left(\frac{\partial}{\partial x_{i}} \frac{F_{i}(\boldsymbol{x})}{\gamma_{i}} \varphi\right)+\frac{T}{\gamma_{i}} \frac{\partial^{2}}{\partial x_{i}^{2}} \varphi\right. \\
& \left.-\frac{F_{i}(\boldsymbol{x})}{\gamma_{i}} \frac{\partial}{\partial X_{i}} \varphi+\frac{T}{\gamma_{i}} \frac{\partial^{2}}{\partial X_{i}^{2}} \varphi+\frac{2 T}{\gamma_{i}} \frac{\partial^{2}}{\partial x_{i} \partial X_{i}} \varphi\right] .
\end{aligned}
$$

Here, we consider a quantity

$$
q_{\boldsymbol{h}}(\boldsymbol{x}, t)=\int_{\mathbf{R}^{N d}} d \boldsymbol{X} e^{\sum_{i=1}^{N d} h_{i} X_{i}} p(\boldsymbol{x}, \boldsymbol{X}, t),
$$

which obeys

$$
\frac{\partial q_{\boldsymbol{h}}}{\partial t}=\mathcal{L}_{\boldsymbol{h}}^{(\boldsymbol{x})} \cdot q_{\boldsymbol{h}},
$$

where the operator $\mathcal{L}_{\boldsymbol{h}}^{(\boldsymbol{x})}$ is calculated as

$$
\begin{aligned}
\mathcal{L}_{\boldsymbol{h}}^{(\boldsymbol{x})} \cdot \varphi & =\sum_{i=1}^{N d}\left[-\left(\frac{\partial}{\partial x_{i}} \frac{F_{i}(\boldsymbol{x})}{\gamma_{i}} \varphi\right)+\frac{T}{\gamma_{i}} \frac{\partial^{2}}{\partial x_{i}^{2}} \varphi\right. \\
& \left.+\frac{F_{i}(\boldsymbol{x})}{\gamma_{i}} h_{i} \varphi+\frac{T}{\gamma_{i}} h_{i}^{2} \varphi-\frac{2 T}{\gamma_{i}} h_{i} \frac{\partial}{\partial x_{i}} \varphi\right] .
\end{aligned}
$$

Then, from (38) and (42), we have

$$
\left\langle e^{t \sum_{i=1}^{N d} h_{i} V_{i}(t)}\right\rangle=\int_{0}^{L} d \boldsymbol{x} q_{\boldsymbol{h}}(\boldsymbol{x}, t) .
$$

Let $\mu_{0}^{h}$ be the largest eigenvalue of $\mathcal{L}_{\boldsymbol{h}}^{(\boldsymbol{x})}$. (43) and (45) lead to the asymptotic form

$$
\left\langle e^{t \sum_{i=1}^{N d} h_{i} V_{i}(t)}\right\rangle \simeq e^{t \mu_{0}^{h}}
$$

when $t$ is sufficiently large. Thus, from the definition of $G^{\boldsymbol{F}}(\boldsymbol{h})$ given in (14), we obtain

$$
G^{\boldsymbol{F}}(\boldsymbol{h})=\mu_{0}^{\boldsymbol{h}} .
$$

The result that a scaled cumulant generating function is equal to the largest eigenvalue of an operator is standard [2]. The fluctuation theorem was studied with the aid of this result [14]. Recently, a relation similar to (47) was employed for calculations of the large deviation function of entropy production [46], where a kink in the large deviation function has been pointed out. See Ref. [47] for a recent related result.

We denote the adjoint operator of $\mathcal{L}_{\boldsymbol{h}}^{(\boldsymbol{x})}$ by $\mathcal{L}_{\boldsymbol{h}}^{(\boldsymbol{x}) \dagger}$, which is calculated as

$$
\begin{aligned}
\mathcal{L}_{\boldsymbol{h}}^{(\boldsymbol{x}) \dagger} \cdot \varphi & =\sum_{i=1}^{N d}\left[\frac{F_{i}(\boldsymbol{x})}{\gamma_{i}} \frac{\partial}{\partial x_{i}} \varphi+\frac{T}{\gamma_{i}} \frac{\partial^{2}}{\partial x_{i}^{2}} \varphi\right. \\
& \left.+\frac{F_{i}(\boldsymbol{x})}{\gamma_{i}} h_{i} \varphi+\frac{T}{\gamma_{i}} h_{i}^{2} \varphi+\frac{2 T}{\gamma_{i}} h_{i} \frac{\partial}{\partial x_{i}} \varphi\right] .
\end{aligned}
$$

Let $\mu_{n}^{\boldsymbol{h}}$ and $\eta_{n}^{\boldsymbol{h}}(n=0,1,2, \ldots)$ be all the eigenvalues of $\mathcal{L}_{h}^{(x)}$ and $\mathcal{L}_{h}^{(\boldsymbol{x}) \dagger}$, respectively. We label the eigenvalues 
such that $\operatorname{Re}\left(\mu_{n}^{\boldsymbol{h}}\right) \geq \operatorname{Re}\left(\mu_{m}^{\boldsymbol{h}}\right)$ and $\operatorname{Re}\left(\eta_{n}^{\boldsymbol{h}}\right) \geq \operatorname{Re}\left(\eta_{m}^{\boldsymbol{h}}\right)$ for $n<m$, where $\operatorname{Re}(\varphi)$ represents the real part of $\varphi$. We may set in general

$$
\mu_{n}^{\boldsymbol{h}}=\left(\eta_{n}^{\boldsymbol{h}}\right)^{*}
$$

with $n=0,1,2, \ldots$, where $\varphi^{*}$ represents the complex conjugate of $\varphi$. As shown in Appendix C, the largest eigenvalues $\mu_{0}^{h}$ and $\eta_{0}^{h}$ are real, and thus

$$
\mu_{0}^{h}=\eta_{0}^{h} .
$$

Furthermore, the eigenfunctions of the largest eigenvalue are positive. From (47) and (50), we obtain

$$
\mathcal{L}_{\boldsymbol{h}}^{(\boldsymbol{x}) \dagger} \cdot \psi_{\boldsymbol{h}}=G^{\boldsymbol{F}}(\boldsymbol{h}) \psi_{\boldsymbol{h}},
$$

where $\psi_{\boldsymbol{h}}(\boldsymbol{x})$ is the positive eigenfunction corresponding to the largest eigenvalue $\eta_{0}^{\boldsymbol{h}}$. Here, by noting $\log \psi_{\boldsymbol{h}}(\boldsymbol{x}) \in$ $\mathbf{R}$, we define a force $\boldsymbol{u}_{\boldsymbol{h}}^{\boldsymbol{F}}(\boldsymbol{x})$ as

$$
u_{\boldsymbol{h}, i}^{\boldsymbol{F}}(\boldsymbol{x}) \equiv 2 T\left(\frac{\partial}{\partial x_{i}} \log \psi_{\boldsymbol{h}}(\boldsymbol{x})+h_{i}\right),
$$

which corresponds to the Cole-Hopf transformation [51]. By using trivial identities

$$
\begin{gathered}
\frac{\partial \psi_{\boldsymbol{h}}(\boldsymbol{x})}{\partial x_{i}}=\psi_{\boldsymbol{h}}(\boldsymbol{x}) \frac{\partial \log \psi_{\boldsymbol{h}}(\boldsymbol{x})}{\partial x_{i}}, \\
\frac{\partial^{2} \psi_{\boldsymbol{h}}(\boldsymbol{x})}{\partial x_{i}^{2}}=\psi_{\boldsymbol{h}}(\boldsymbol{x})\left\{\frac{\partial^{2} \log \psi_{\boldsymbol{h}}(\boldsymbol{x})}{\partial x_{i}^{2}}+\left(\frac{\partial \log \psi_{\boldsymbol{h}}(\boldsymbol{x})}{\partial x_{i}}\right)^{2}\right\},
\end{gathered}
$$

we rewrite (51) as

$$
\begin{aligned}
G^{\boldsymbol{F}}(\boldsymbol{h})=\frac{1}{2 T} & \sum_{i=1}^{N d}\left[\frac{F_{i}(\boldsymbol{x})}{\gamma_{i}} u_{\boldsymbol{h}, i}^{\boldsymbol{F}}(\boldsymbol{x})\right. \\
& \left.+\frac{1}{2 \gamma_{i}} u_{\boldsymbol{h}, i}^{\boldsymbol{F}}(\boldsymbol{x})^{2}+\frac{T}{\gamma_{i}} \frac{\partial}{\partial x_{i}} u_{\boldsymbol{h}, i}^{\boldsymbol{F}}(\boldsymbol{x})\right] .
\end{aligned}
$$

Now, we consider a modified system with an arbitrary external force $\boldsymbol{w}(\boldsymbol{x})$ added. The stationary probability density in the modified system, $P_{\mathrm{st}}^{\boldsymbol{F}+\boldsymbol{w}}(\boldsymbol{x})$, satisfies (11) and (12) with the replacement of $\boldsymbol{F}$ by $\boldsymbol{F}+\boldsymbol{w}$. By multiplying (55) by $P_{\mathrm{st}}^{\boldsymbol{F}+\boldsymbol{w}}(\boldsymbol{x})$, integrating it with respect to $\boldsymbol{x}$, and integrating by parts in the third term, we obtain

$$
\begin{aligned}
G^{\boldsymbol{F}}(\boldsymbol{h})=\frac{1}{2 T} & \int_{\Omega^{N}} d \boldsymbol{x} \sum_{i=1}^{N d} u_{\boldsymbol{h}, i}^{\boldsymbol{F}}(\boldsymbol{x})\left[P_{\mathrm{st}}^{\boldsymbol{F}+\boldsymbol{w}}(\boldsymbol{x}) \frac{F_{i}(\boldsymbol{x})}{\gamma_{i}}\right. \\
& \left.+P_{\mathrm{st}}^{\boldsymbol{F}+\boldsymbol{w}}(\boldsymbol{x}) \frac{1}{2 \gamma_{i}} u_{\boldsymbol{h}, i}^{\boldsymbol{F}}(\boldsymbol{x})-\frac{T}{\gamma_{i}} \frac{\partial P_{\mathrm{st}}^{\boldsymbol{F}+\boldsymbol{w}}(\boldsymbol{x})}{\partial x_{i}}\right] .
\end{aligned}
$$

Then, we substitute (11) into (56) and we replace $P_{\mathrm{st}}^{\boldsymbol{F}+\boldsymbol{w}}(\boldsymbol{x}) F_{i}(\boldsymbol{x}) / \gamma_{i}-\left(T / \gamma_{i}\right) \partial P_{\mathrm{st}}^{\boldsymbol{F}+\boldsymbol{w}} / \partial x_{i}$ by $J_{\mathrm{st}, i}^{\boldsymbol{F}+\boldsymbol{w}}(\boldsymbol{x})-$
$P_{\text {st }}^{\boldsymbol{F}}(\boldsymbol{x}) w_{i}(\boldsymbol{x}) / \gamma_{i}$. Furthermore, by substituting (52) into the term $u_{\boldsymbol{h}, i}^{\boldsymbol{F}}(\boldsymbol{x}) J_{\mathrm{st}, i}^{\boldsymbol{F}+\boldsymbol{w}}(\boldsymbol{x})$, we rewrite (56) as

$$
\begin{aligned}
& G^{\boldsymbol{F}}(\boldsymbol{h})=\int_{\Omega^{N}} d \boldsymbol{x} \sum_{i=1}^{N d}\left[h_{i} J_{\mathrm{st}, i}^{\boldsymbol{F}+\boldsymbol{w}}(\boldsymbol{x})-\frac{P_{\mathrm{st}}^{\boldsymbol{F}+\boldsymbol{w}}(\boldsymbol{x})}{4 T \gamma_{i}} w_{i}(\boldsymbol{x})^{2}\right] \\
& +\int_{\Omega^{N}} d \boldsymbol{x} \sum_{i=1}^{N d} \frac{P_{\mathrm{st}}^{\boldsymbol{F}+\boldsymbol{w}}(\boldsymbol{x})}{4 T \gamma_{i}}\left(u_{\boldsymbol{h}, i}^{\boldsymbol{F}}(\boldsymbol{x})-w_{i}(\boldsymbol{x})\right)^{2},
\end{aligned}
$$

where we have used (12). Finally, by recalling (19) and (25), we arrive at

$$
G^{\boldsymbol{F}}(\boldsymbol{h})=\Phi_{\boldsymbol{h}}^{\boldsymbol{F}}(\boldsymbol{w})+\sum_{i=1}^{N d} \frac{1}{4 T \gamma_{i}}\left\langle\left(u_{\boldsymbol{h}, i}^{\boldsymbol{F}}-w_{i}\right)^{2}\right\rangle_{\mathrm{st}}^{\boldsymbol{F}+\boldsymbol{w}}
$$

It should be noted that the external force $\boldsymbol{w}(\boldsymbol{x})$ in (58) is arbitrary.

Here, the second term of the right-hand side in (58) is not negative and becomes zero only when $\boldsymbol{w}=\boldsymbol{u}_{\boldsymbol{h}}^{\boldsymbol{F}}$. This leads to

$$
G^{\boldsymbol{F}}(\boldsymbol{h})=\Phi_{\boldsymbol{h}}^{\boldsymbol{F}}\left(\boldsymbol{u}_{\boldsymbol{h}}^{\boldsymbol{F}}\right) \geq \Phi_{\boldsymbol{h}}^{\boldsymbol{F}}(\boldsymbol{w}),
$$

where the equality holds only when $\boldsymbol{w}=\boldsymbol{u}_{\boldsymbol{h}}^{\boldsymbol{F}}$. This result is the variational expression (26). The optimal force $\boldsymbol{w}_{\boldsymbol{h}}^{\boldsymbol{F} \text {,opt }}(\boldsymbol{x})$ defined by (27) is now derived as

$$
\boldsymbol{w}_{\boldsymbol{h}}^{\boldsymbol{F}, \mathrm{opt}}(\boldsymbol{x})=\boldsymbol{u}_{\boldsymbol{h}}^{\boldsymbol{F}}(\boldsymbol{x}) .
$$

Next, the derivative of (58) with respect to $h_{i}$ yields

$$
\begin{aligned}
& \frac{\partial G^{\boldsymbol{F}}(\boldsymbol{h})}{\partial h_{i}} \\
& =\left\langle V_{i}\right\rangle_{\mathrm{st}}^{\boldsymbol{F}+\boldsymbol{w}}+\sum_{i=1}^{N d}\left[\frac{1}{2 T \gamma_{i}}\left\langle\left(u_{\boldsymbol{h}, i}^{\boldsymbol{F}}-w_{i}\right) \frac{\partial u_{\boldsymbol{h}, i}^{\boldsymbol{F}}}{\partial h_{i}}\right\rangle_{\mathrm{st}}^{\boldsymbol{F}+\boldsymbol{w}}\right] .
\end{aligned}
$$

Since $\boldsymbol{w}$ is arbitrary, we may set $\boldsymbol{w}=\boldsymbol{w}_{\boldsymbol{h}}^{\boldsymbol{F} \text {,opt }}$ in the expression. This provides the result (28). Furthermore, from (52) and (60), we obtain (29) with (30), because the eigenfunction $\psi_{\boldsymbol{h}}(\boldsymbol{x})$ satisfies $\lim _{\boldsymbol{h} \rightarrow 0} \psi_{\boldsymbol{h}}(\boldsymbol{x})=$ const.

\section{REMARKS ON THE VARIATIONAL FUNCTION $\Phi_{h}^{F}$}

In this section, we present three remarks on the variational function $\Phi_{\boldsymbol{h}}^{\boldsymbol{F}}(\boldsymbol{w})$. Before entering the main part, we first show a slightly different expression of $\Phi_{\boldsymbol{h}}^{\boldsymbol{F}}(\boldsymbol{w})$. Since we have (29), we may restrict $\boldsymbol{w}(\boldsymbol{x})$ to the form

$$
\boldsymbol{w}(\boldsymbol{x})=2 T \boldsymbol{h}-\frac{\partial}{\partial \boldsymbol{x}} V(\boldsymbol{x}) .
$$

Throughout this section, the extra force $\boldsymbol{w}(\boldsymbol{x})$ is always connected to the potential function $V(\boldsymbol{x})$ through (62). We then rewrite (26) as

$$
G^{\boldsymbol{F}}(\boldsymbol{h})=\max _{V} \Phi_{\boldsymbol{h}}^{\boldsymbol{F}}(\boldsymbol{w}),
$$


where the optimal potential $V_{\boldsymbol{h}}^{\boldsymbol{F}, \text { opt }}$ satisfies

$$
\boldsymbol{w}_{\boldsymbol{h}}^{\boldsymbol{F}, \mathrm{opt}}(\boldsymbol{x})=2 T \boldsymbol{h}-\frac{\partial}{\partial \boldsymbol{x}} V_{\boldsymbol{h}}^{\boldsymbol{F}, \mathrm{opt}}(\boldsymbol{x}) .
$$

Furthermore, by noting

$$
\int_{\Omega^{N}} d \boldsymbol{x} \sum_{i=1}^{N d} 2 T h_{i} J_{\mathrm{st}, i}^{\boldsymbol{F}+\boldsymbol{w}}(\boldsymbol{x})=\int_{\Omega^{N}} d \boldsymbol{x} \sum_{i=1}^{N d} w_{i}(\boldsymbol{x}) J_{\mathrm{st}, i}^{\boldsymbol{F}+\boldsymbol{w}}(\boldsymbol{x}),
$$

and by using (19), we express $\Phi_{\boldsymbol{h}}^{\boldsymbol{F}}(\boldsymbol{w})$ as

$$
\begin{aligned}
\Phi_{\boldsymbol{h}}^{\boldsymbol{F}}(\boldsymbol{w})=\frac{1}{4 T} \int_{\Omega^{N}} d \boldsymbol{x} \sum_{i=1}^{N d} & {\left[2 J_{\mathrm{st}, i}^{\boldsymbol{F}+\boldsymbol{w}}(\boldsymbol{x}) w_{i}(\boldsymbol{x})\right.} \\
& \left.-\frac{P_{\mathrm{st}}^{\boldsymbol{F}+\boldsymbol{w}}(\boldsymbol{x})}{\gamma_{i}} w_{i}(\boldsymbol{x})^{2}\right] .
\end{aligned}
$$

The expression of (63) with (66) was reported in Ref. 35].

This section is organized as follows. In section IVA, we rewrite the variational function (66) as the difference between two entropy production rates. In section IVB, we point out a similarity between the Donsker-Varadhan formula and our formula. Finally, in section IVC, we show that the cumulant generating function of time-averaged current in a simple electric circuit is expressed in terms of the least energy dissipation rate associated with a variational principle that determines the voltage distribution.

\section{A. Expression of $\Phi_{h}^{F}$ in terms of entropy production rate}

In this subsection, we express the variational function (66) in terms of entropy production rate. First, the probability current for a given probability density $P(\boldsymbol{x})$ is written as

$$
q_{i}^{\boldsymbol{F}}(\boldsymbol{x} \mid P)=\frac{F_{i}(\boldsymbol{x})}{\gamma_{i}} P(\boldsymbol{x})-\frac{T}{\gamma_{i}} \frac{\partial P(\boldsymbol{x})}{\partial x_{i}},
$$

where the slightly heavy notation $q_{i}^{\boldsymbol{F}}(\boldsymbol{x} \mid P)$ is used in order to distinguish $\boldsymbol{F}$ and $P$ dependence in the current. Here, we define the functional $\sigma^{\boldsymbol{F}}(P)$ as

$$
\sigma^{\boldsymbol{F}}(P) \equiv \frac{1}{T} \int_{\Omega^{N}} d \boldsymbol{x} \sum_{i=1}^{N d} \frac{\gamma_{i}}{P(\boldsymbol{x})}\left(q_{i}^{\boldsymbol{F}}(\boldsymbol{x} \mid P)\right)^{2} .
$$

By substituting (67) into (68), we express $\sigma^{\boldsymbol{F}}(P)$ as

$$
\begin{aligned}
& \sigma^{\boldsymbol{F}}(P) \\
& =\int_{\Omega^{N}} d \boldsymbol{x} \sum_{i=1}^{N d} q_{i}^{\boldsymbol{F}}(\boldsymbol{x} \mid P)\left(-\frac{\partial}{\partial x_{i}} \log P(\boldsymbol{x})+\frac{1}{T} F_{i}(\boldsymbol{x})\right) .
\end{aligned}
$$

The integration by parts of the first term leads to

$$
\begin{aligned}
\sigma^{\boldsymbol{F}}(P)= & \int_{\Omega^{N}} d \boldsymbol{x} \log P(\boldsymbol{x}) \sum_{i=1}^{N d} \frac{\partial}{\partial x_{i}} q_{i}^{\boldsymbol{F}}(\boldsymbol{x} \mid P) \\
& +\frac{1}{T} \int_{\Omega^{N}} d \boldsymbol{x} \sum_{i=1}^{N d} q_{i}^{\boldsymbol{F}}(\boldsymbol{x} \mid P) F_{i}(\boldsymbol{x}) .
\end{aligned}
$$

We denote the first and second terms of the right-hand side in (70) by $\Sigma^{\boldsymbol{F}}(P)$ and $\Omega^{\boldsymbol{F}}(P)$, respectively. Then, $\Sigma^{\boldsymbol{F}}(P)$ is equal to the increasing rate of the Shannon entropy and $\Omega^{\boldsymbol{F}}(P)$ corresponds to the energy-dissipation rate divided by $T$. See Appendix $\mathrm{C}$ for a more detailed explanation. Since $\Omega^{\boldsymbol{F}}(P)$ is identical to the increasing rate of the thermodynamic entropy of the heat bath, we identify $\sigma^{\boldsymbol{F}}(P)$ with entropy production rate of the total system.

Now, from (67) and (68), we calculate

$$
\begin{array}{r}
\sigma^{\boldsymbol{F}+\boldsymbol{w}}\left(P_{\mathrm{st}}^{\boldsymbol{F}+\boldsymbol{w}}\right)-\sigma^{\boldsymbol{F}}\left(P_{\mathrm{st}}^{\boldsymbol{F}+\boldsymbol{w}}\right) \\
=\frac{1}{T} \int_{\Omega^{N}} d \boldsymbol{x} \sum_{i=1}^{N d}\left[2 q_{i}^{\boldsymbol{F}+\boldsymbol{w}}\left(\boldsymbol{x} \mid P_{\mathrm{st}}^{\boldsymbol{F}+\boldsymbol{w}}\right) w_{i}(\boldsymbol{x})\right. \\
\left.-\frac{P_{\mathrm{st}}^{\boldsymbol{F}+\boldsymbol{w}}(\boldsymbol{x})}{\gamma_{i}} w_{i}(\boldsymbol{x})^{2}\right] .
\end{array}
$$

By noting $q_{i}^{\boldsymbol{F}+\boldsymbol{w}}\left(\boldsymbol{x} \mid P_{\mathrm{st}}^{\boldsymbol{F}+\boldsymbol{w}}\right)=J_{\mathrm{st}, i}^{\boldsymbol{F}+\boldsymbol{w}}(\boldsymbol{x})$ and comparing (71) with (66), we obtain

$$
\Phi_{\boldsymbol{h}}^{\boldsymbol{F}}(\boldsymbol{w})=\frac{1}{4}\left[\sigma^{\boldsymbol{F}+\boldsymbol{w}}\left(P_{\mathrm{st}}^{\boldsymbol{F}+\boldsymbol{w}}\right)-\sigma^{\boldsymbol{F}}\left(P_{\mathrm{st}}^{\boldsymbol{F}+\boldsymbol{w}}\right)\right] .
$$

The first term of the right-hand side in (72) is proportional to the entropy production rate of the modified system in the steady state. The second term of (72) is a little bit complicated. $\sigma^{\boldsymbol{F}}\left(P_{\mathrm{st}}^{\boldsymbol{F}+\boldsymbol{w}}\right)$ is the entropy production rate just after the extra force $\boldsymbol{w}$ is turned off suddenly in the steady state of the modified system. In this manner, the expression (72) provides us a thermodynamic interpretation of the variational function $\Phi_{h}^{F}$.

\section{B. Similarity with the Donsker-Varadhan formula}

For a given path $(\boldsymbol{x}(t))_{t=0}^{\tau}$, we define the occupation density as

$$
P_{\tau}^{\mathrm{em}}(\boldsymbol{x}) \equiv \frac{1}{\tau} \int_{0}^{\tau} d t \delta(\boldsymbol{x}(t)-\boldsymbol{x}) .
$$

We denote the probability density of $\left(P_{\tau}^{\mathrm{em}}(\boldsymbol{x})\right)_{\boldsymbol{x} \in \Omega}$ by $\operatorname{Prob}_{\tau}^{\boldsymbol{F}}(\mathrm{P})$. We write it formally as

$$
\operatorname{Prob}_{\tau}^{\boldsymbol{F}}(P)=\left\langle\prod_{\boldsymbol{x} \in \Omega} \delta\left(P_{\tau}^{\mathrm{em}}(\boldsymbol{x})-P(\boldsymbol{x})\right)\right\rangle .
$$

When $\tau$ is sufficiently large, the asymptotic form of $\operatorname{Prob}_{\tau}^{\boldsymbol{F}}(P)$ is given by

$$
\operatorname{Prob}_{\tau}^{\boldsymbol{F}}(P) \simeq e^{-\tau I^{\boldsymbol{F}}(P)},
$$


which is the large deviation property of the occupation density with the large deviation function $I^{\boldsymbol{F}}(P)$. Let $\mathcal{L}_{\mathrm{FP}}^{(\boldsymbol{x}) \dagger}$ be the adjoint Fokker-Planck operator defined by

$$
\mathcal{L}_{\mathrm{FP}}^{(\boldsymbol{x}) \dagger} \cdot \varphi=\sum_{i=1}^{N d}\left[\frac{F_{i}(\boldsymbol{x})}{\gamma_{i}} \frac{\partial}{\partial x_{i}} \varphi+\frac{T}{\gamma_{i}} \frac{\partial^{2}}{\partial x_{i}^{2}} \varphi\right] .
$$

Then, the Donsker-Varadhan formula in the Langevin system is written as

$$
I^{\boldsymbol{F}}(P)=-\min _{\phi>0} \int_{\Omega^{N}} d \boldsymbol{x} P(\boldsymbol{x}) \frac{\mathcal{L}_{\mathrm{FP}}^{(\boldsymbol{x}) \dagger} \cdot \phi}{\phi(\boldsymbol{x})} .
$$

Such a formula was rigorously derived for general Markov stochastic processes [52]. Its universal form suggests that it might be connected to a framework of non-equilibrium statistical mechanics. Indeed, in Ref. [53], it has been pointed out that the minimum entropy production principle is related to the Donsker-Varadhan formula (77). This relation was obtained by noting the detailed balance condition in equilibrium systems. Similarly, in equilibrium dynamics, the variational principle determining the activity was recognized as a form similar to the DonskerVaradhan formula [54]. More recently, even without the detailed balance condition, the variational function in the Donsker-Varadhan formula was conjectured to be related to the activity [55].

Based on these achievements, we rewrite (77) so as to see a similarity with our variational function (72). First, since $\phi$ in (77) is positive, we define the potential $V(\boldsymbol{x})$ by

$$
V(\boldsymbol{x}) \equiv-2 T \log \phi(\boldsymbol{x}) .
$$

Then, using (53) and (54), we rewrite (77) as

$$
\begin{aligned}
& I^{\boldsymbol{F}}(P)=-\frac{1}{2 T} \min _{\phi(\boldsymbol{x})>0} \int_{\Omega^{N}} d \boldsymbol{x} P(\boldsymbol{x}) \sum_{i=1}^{N d} \\
& {\left[-\frac{F_{i}(\boldsymbol{x})}{\gamma_{i}} \frac{\partial V(\boldsymbol{x})}{\partial x_{i}}-\frac{T}{\gamma_{i}} \frac{\partial^{2} V(\boldsymbol{x})}{\partial x_{i}^{2}}+\frac{1}{2 \gamma_{i}}\left(-\frac{\partial V(\boldsymbol{x})}{\partial x_{i}}\right)^{2}\right] .}
\end{aligned}
$$

The integration by parts in the second term leads to

$$
\begin{aligned}
& I^{\boldsymbol{F}}(P)=-\frac{1}{2 T} \min _{\phi(\boldsymbol{x})>0} \int_{\Omega^{N}} d \boldsymbol{x} \sum_{i=1}^{N d}\left(-\frac{\partial V(\boldsymbol{x})}{\partial x_{i}}\right) \\
& {\left[\frac{F_{i}(\boldsymbol{x})}{\gamma_{i}} P(\boldsymbol{x})-\frac{T}{\gamma_{i}} \frac{\partial P(\boldsymbol{x})}{\partial x_{i}}+\frac{1}{2 \gamma_{i}}\left(-\frac{\partial V(\boldsymbol{x})}{\partial x_{i}}\right) P(\boldsymbol{x})\right] .}
\end{aligned}
$$

Thus, by using (67) with the replacement of $\boldsymbol{F}$ by $\boldsymbol{F}-$ $\partial V / \partial \boldsymbol{x}$, we obtain

$$
\begin{aligned}
& I^{\boldsymbol{F}}(P)=-\frac{1}{4 T} \min _{V(\boldsymbol{x})} \int_{\Omega^{N}} d \boldsymbol{x} \sum_{i=1}^{N d} \\
& {\left[2\left(-\frac{\partial V(\boldsymbol{x})}{\partial x_{i}}\right) q_{i}^{\boldsymbol{F}-\partial V / \partial \boldsymbol{x}}(\boldsymbol{x} \mid P)-\frac{1}{\gamma_{i}}\left(-\frac{\partial V(\boldsymbol{x})}{\partial x_{i}}\right)^{2} P(\boldsymbol{x})\right] .}
\end{aligned}
$$

Furthermore, by comparing (81) with (71), we find

$$
I^{\boldsymbol{F}}(P)=-\frac{1}{4} \min _{V} \int_{\Omega^{N}} d \boldsymbol{x}\left[\sigma^{\boldsymbol{F}-\partial V / \partial \boldsymbol{x}}(P)-\sigma^{\boldsymbol{F}}(P)\right] .
$$

These expressions, (81) and (82), were derived in Ref. [56, 57] by using another method. For the large deviation function of occupation density in jump processes, a similar expression in terms of escape rates instead of the entropy production rates has been reported in Ref. [55].

It is seen that there is a strong similarity between (82) and (63) with (72). This suggests that two formulas might be described in a unified manner. We will report a unified treatment of the Donsker-Varadhan formula and ours in another paper.

\section{Relationship to the principle of the least dissipation of energy}

We consider an electric circuit in which $n$ resistances $\left(R_{1}, R_{2}, \ldots, R_{n}\right)$ are connected in series. We impose an electric potential $V$ on the circuit by using a battery as shown in the left side of Fig. 1,

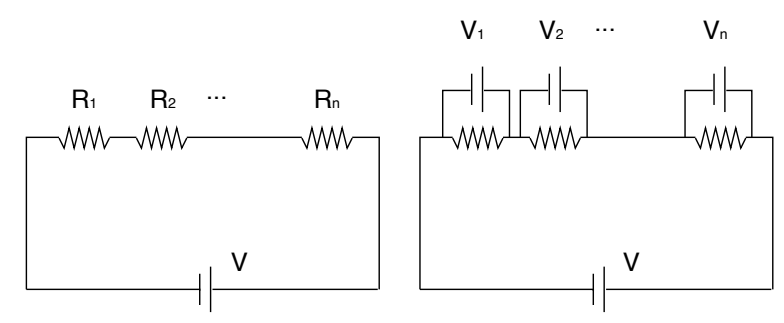

FIG. 1: Left: $n$ resistances $\left(R_{1}, R_{2}, \ldots, R_{n}\right)$ are connected in series. We impose an electric potential $V$ on the circuit. Right: By using $n$ batteries, we impose an electric potential $V_{i}$ on each resistance $R_{i}$.

Let $V_{* i}$ be the difference of the electric potential at the resistance $R_{i}$ in the electric circuit. This $V_{* i}$ is determined by Ohm's law

$$
V_{* i}=I_{*} R_{i},
$$

where $I_{*}$ is the electric current in the circuit. From the constraint condition $\sum_{i=1}^{n} V_{* i}=V$ and Ohm's law (83), we obtain

$$
\begin{gathered}
I_{*}=\frac{V}{\sum_{i=1}^{n} R_{i}}, \\
V_{* i}=V \frac{R_{i}}{\sum_{j=1}^{n} R_{j}} .
\end{gathered}
$$

The principle of the least dissipation of energy claims that $V_{* i}$ is characterized by the least dissipation of energy [58]. In order to see this statement, we consider an 
electric circuit displayed in the right side of Fig. 1, By using $n$ batteries, we impose an electric potential $V_{i}$ on each resistance $R_{i}$ such that $\sum_{i=1}^{n} V_{i}=V$. Under the assumption that the energy dissipation is determined by the Joule heating, the energy dissipation rate $K$ is calculated as

$$
K\left(V_{1}, V_{2}, \ldots, V_{n}\right)=\sum_{i=1}^{n} \frac{V_{i}^{2}}{R_{i}} .
$$

Then, one can directly confirm that $V_{* i}$ is determined as

$$
\left(V_{* i}\right)_{i=1}^{n}=\underset{\substack{\left(V_{i}\right)_{i=1}^{n} \\\left(\sum_{i} V_{i}=V\right)}}{\operatorname{argmin}} K\left(V_{1}, V_{2}, \ldots, V_{n}\right),
$$

where $\left(\sum_{i=1}^{n} V_{i}=V\right)$ means the constraint condition for $\left(V_{i}\right)_{i=1}^{n}$. This is an example of the principle of the least dissipation of energy. We define the least energy dissipation rate $K_{*}(V)$ as

$$
K_{*}(V)=\min _{\substack{\left(V_{i}\right)_{i=1}^{n} \\\left(\sum_{i} V_{i}=V\right)}} K\left(V_{1}, V_{2}, \ldots, V_{n}\right) .
$$

Next, we consider fluctuations of the time-averaged electric current $I$ during a time interval $[0, \tau]$. In a manner similar to (14), we define the scaled cumulant generating function of the time-averaged current as

$$
G(h) \equiv \lim _{\tau \rightarrow \infty} \frac{1}{\tau} \log \left\langle e^{\tau I h}\right\rangle .
$$

For simplicity, we assume that the current fluctuations are described by Johnson noise. See the first chapter of Ref. [36] for a brief review of Johnson noise. The Johnson noise satisfies the fluctuation dissipation relation

$$
\lim _{\tau \rightarrow \infty} \tau\left(\left\langle I^{2}\right\rangle-\langle I\rangle^{2}\right)=\frac{2 T}{\sum_{i} R_{i}} .
$$

We also assume that the fluctuations are Gaussian. Under these assumptions, we write $G(h)$ as

$$
G(h)=\frac{V}{\sum_{i=1}^{n} R_{i}} h+\frac{T}{\sum_{i=1}^{n} R_{i}} h^{2} .
$$

Now, we connect the least energy dissipation rate $K_{*}(V)$ and the cumulant generating function $G(h)$. First, from (85) and (88), we find

$$
K_{*}(V)=\frac{V^{2}}{\sum_{j} R_{j}} .
$$

Then, by replacing $V$ by $V+2 T h$ in (92), we obtain

$$
K_{*}(V+2 T h)=\frac{V^{2}}{\sum_{j} R_{j}}+4 T G(h),
$$

where we have used (91). The subtraction of (92) from (93) leads to

$$
G(h)=\frac{1}{4 T}\left[K_{*}(V+2 T h)-K_{*}(V)\right] .
$$

This result that $G(h)$ is written by the difference of the energy dissipation rates for two different states reminds us of $\mathrm{A} 8 \mathrm{C}$ in equilibrium statistical mechanics. Our formula (63) with (72) is a generalization of (94).

\section{APPLICATIONS}

In this section, we present three applications of our result by restricting our investigation to a Brownian particle on a ring. We write an explicit setting. Let $x(t)$ be a position of the particle along the ring of size $L$. We assume that the motion of the particle is described by

$$
\dot{x}=\frac{1}{\gamma} F(x)+\sqrt{\frac{2 T}{\gamma}} \xi
$$

with the periodic boundary condition, where $F(x)$ is a deterministic force given by

$$
F(x)=f-\frac{\partial U(x)}{\partial x} .
$$

Such a force can be realized by using a rotating periodic potential [41, 42]. Note that (95) with (96) corresponds to the case that $N=d=1$ in the model studied in Section II] The stationary distribution $P_{\text {st }}^{F}(x)$ and the stationary current $J_{\text {st }}^{F}$ are determined by

$$
J_{\mathrm{st}}^{F}=\frac{1}{\gamma} F(x) P_{\mathrm{st}}^{F}(x)-\frac{T}{\gamma} \frac{\partial}{\partial x} P_{\mathrm{st}}^{F}(x)
$$

with the periodic boundary condition $P_{\mathrm{st}}^{F}(0)=P_{\mathrm{st}}^{F}(L)$ and the normalization condition $\int_{0}^{L} d x P_{\mathrm{st}}^{F}(x)=1$.

For this system, in section $\mathrm{VA}$, we derive the diffusion constant $D=\lim _{\tau \rightarrow \infty}(1 / 2) \tau\left\langle V(\tau)^{2}\right\rangle_{c}$ and the mobility $\mu=(\partial / \partial f) \lim _{\tau \rightarrow \infty}\langle V(\tau)\rangle_{c}$ by utilizing our formula. Then, in section $\mathrm{VB}$, we show that our variational expression (26) with (25) leads to a formula derived from an additivity principle [25 28]. Finally, in section $\mathrm{VC}$, we present an experimental method for determining the cumulant generating function without the information of $F(x)$.

\section{A. Diffusion constant and mobility}

In the system under study, the stationary distribution $P_{\text {st }}^{F}(x)$, the diffusion constant $D$ and the mobility $\mu$ are known to be expressed in terms of the following functions 39, 40, 59]:

$$
I_{ \pm}(x) \equiv \int_{0}^{L} d y e^{ \pm \int_{x}^{x \mp y} d z F(z) / T} .
$$

Concretely, the expressions are

$$
\begin{gathered}
P_{\mathrm{st}}^{F}(x)=\alpha I_{-}(x), \\
D=\frac{T}{\gamma} L^{2} \alpha^{3} \int_{0}^{L} d x I_{+}(x) I_{-}(x)^{2},
\end{gathered}
$$

and

$$
\mu=\frac{L}{\gamma} \alpha^{2} \int_{0}^{L} d x I_{+}(x) I_{-}(x)
$$


where $\alpha$ is the normalization constant given by

$$
\alpha \equiv\left(\int_{0}^{L} d x I_{ \pm}(x)\right)^{-1} .
$$

Here, one may wonder about the origin of such compact expressions. Below, within our formulation, we uncover a simple structure behind the expressions.

First, we consider the diffusion constant. We start with the formula (58) with (25). In the case that $N=d=1$, it becomes

$$
\begin{aligned}
& G^{F}(h) \\
& =h\langle V\rangle_{\mathrm{st}}^{F+w}-\frac{1}{4 T \gamma}\left[\left\langle w^{2}\right\rangle_{\mathrm{st}}^{F+w}-\left\langle\left(u_{h}^{F}-w\right)^{2}\right\rangle_{\mathrm{st}}^{F+w}\right],
\end{aligned}
$$

where it should be noted that $w(x)$ is arbitrary. By setting $w=0$ in (103), we obtain

$$
G^{F}(h)=h\langle V\rangle_{\mathrm{st}}^{F}+\frac{1}{4 T \gamma}\left\langle\left(u_{h}^{F}\right)^{2}\right\rangle_{\mathrm{st}}^{F} .
$$

Since $\left.u_{h}^{F}(x)\right|_{h=0}=0$ (See (30) and (52)), $u_{h}^{F}(x)$ is expanded as

$$
u_{h}^{F}(x)=\sum_{n=1}^{\infty} u_{n}(x) h^{n} .
$$

The substitution of (105) into (104) yields

$$
G^{F}(h)=h\langle V\rangle_{\mathrm{st}}^{F}+\frac{1}{4 T \gamma}\left\langle\left(u_{1}\right)^{2}\right\rangle_{\mathrm{st}}^{F} h^{2}+\mathcal{O}\left(h^{3}\right),
$$

which leads to

$$
D=\frac{1}{4 T \gamma}\left\langle\left(u_{1}\right)^{2}\right\rangle_{\text {st }}^{F} .
$$

Next, we consider the mobility. Let us fix a function $w^{\prime}(x)$. By setting $w=-F+w^{\prime}$ in (103), we rewrite (103) as

$G^{F}(h)=h\langle V\rangle_{\mathrm{st}}^{w^{\prime}}-\frac{1}{4 T \gamma}\left\langle\left(w^{\prime}-F\right)^{2}-\left(u_{h}^{F}-w^{\prime}+F\right)^{2}\right\rangle_{\mathrm{st}}^{w^{\prime}}$

We then differentiate (108) with respect to force $f$. The result is

$$
\begin{aligned}
& \frac{\partial G^{F}(h)}{\partial f} \\
& =\frac{1}{2 T \gamma}\left\langle\left(w^{\prime}-F\right)+\left(u_{h}^{F}-w^{\prime}+F\right)\left(\frac{\partial u_{h}^{F}}{\partial f}+1\right)\right\rangle_{\mathrm{st}}^{w^{\prime}} .
\end{aligned}
$$

Since $w^{\prime}(x)$ is arbitrary in this expression, we may set $w^{\prime}=u_{h}^{F}+F$. This leads to

$$
\frac{\partial G^{F}(h)}{\partial f}=\frac{1}{2 T \gamma}\left\langle u_{h}^{F}\right\rangle_{\mathrm{st}}^{F+u_{h}^{F}}
$$

By substituting (105), (106) into (110), we obtain

$$
\mu=\frac{1}{2 T \gamma}\left\langle u_{1}\right\rangle_{\mathrm{st}}^{F} .
$$

As shown in (107) and (111), $D$ and $\mu$ are expressed by the second and the first moments of $u_{1}(x)$, respectively. Here, we determine $u_{1}(x)$. We substitute (105) into (55) and extract the terms proportional to $h$. We then obtain

$$
2 T\langle V\rangle_{\mathrm{st}}^{F}=\frac{T}{\gamma} \frac{\partial}{\partial x} u_{1}(x)+\frac{1}{\gamma} F(x) u_{1}(x) .
$$

Note the periodic boundary condition $u_{1}(0)=u_{1}(L)$ and the normalization condition

$$
\int_{0}^{L} d x u_{1}(x)=2 T L
$$

which comes from (52). By comparing (112) with (97), we find that $u_{1}(x)$ can be solved in the manner similar to $P_{\mathrm{st}}^{F}(x)$. The result is

$$
u_{1}(x)=2 T L \alpha I_{+}(x) .
$$

By substituting (114) and (99) into (107) and (111), we obtain (100) and (101). Furthermore, we can systematically evaluate higher-order cumulant coefficients by formulating an iterative relation. See Appendix D.

At the end of this subsection, we study the violation of the fluctuation-dissipation relation. First, from (18), we have

$$
\langle V\rangle_{\mathrm{st}}^{F+u_{h}^{F}}-\frac{1}{\gamma}\langle F\rangle_{\mathrm{st}}^{F+u_{h}^{F}}=\left\langle u_{h}^{F}\right\rangle_{\mathrm{st}}^{F+u_{h}^{F}} .
$$

We substitute (115) into (110) and combine it with our main result (28). We then obtain

$$
\frac{\partial G^{F}(h)}{\partial f}=\frac{1}{2 T} \frac{\partial G^{F}(h)}{\partial h}-\frac{1}{2 T \gamma}\langle F\rangle_{\mathrm{st}}^{F+u_{h}^{F}},
$$

which leads to

$$
\frac{D}{T}-\frac{\partial\langle V\rangle_{\mathrm{st}}^{F}}{\partial f}=\frac{1}{2 T \gamma} \lim _{h \rightarrow 0} \frac{\partial}{\partial h}\langle F\rangle_{\mathrm{st}}^{F+u_{h}^{F}} .
$$

Furthermore, by considering an expansion form

$$
P_{\mathrm{st}}^{F+u_{h}^{F}}(x)=P_{\mathrm{st}}^{F}(x)+h Q_{1}(x)+\mathcal{O}\left(h^{2}\right),
$$

we rewrite the right-hand side of (117) as

$$
\frac{1}{2 T \gamma} \int_{0}^{L} d x F(x) Q_{1}(x)
$$

The expression (119) represents a degree of the violation of the fluctuation-dissipation relation. Recently, several attempts have been presented so as to characterize the violation of fluctuation-dissipation relation $60-64]$. It might be interesting to find a connection of (119) with them. 
When the driving force $f$ is absent, $Q_{1}(x)$ becomes zero so that the fluctuation-dissipation relation holds. We show this fact explicitly. We substitute (118) into (97) with the replacement of $F(x)$ with $F(x)+u_{h}^{F}(x)$, and extract the terms proportional to $h$. We then obtain the following differential equation for $Q_{1}(x)$ :

$$
\frac{\partial}{\partial x}\left[\frac{1}{\gamma} F(x) Q_{1}(x)+\frac{1}{\gamma} u_{1}(x) P_{\mathrm{st}}^{F}(x)-\frac{T}{\gamma} \frac{\partial}{\partial x} Q_{1}(x)\right]=0 .
$$

with the periodic boundary condition $Q_{1}(0)=Q_{1}(L)$ and the normalization condition

$$
\int_{0}^{L} d x Q_{1}(x)=0
$$

which comes from (118). Here, from (99) and (114), we find that $u_{1}(x) P_{\mathrm{st}}^{F}(x)$ is constant in $x$ when $F(x)=$ $-\partial U(x) / \partial x$. In this case, (120) provides us $Q_{1}(x)=$ $C_{1} e^{-U(x) / T}$, where $C_{1}$ is a constant. Then, (121) leads to $C_{1}=0$. Thus, recalling (119), we conclude that the right-hand side of (117) is equal to zero when $f$ is absent. Note that the discussion above applies to many-body systems and higher-dimensional cases.

\section{B. Relation to an additivity principle}

We start with the variational expression (26) with (25). Since the probability current is independent of $x$ in the one-dimensional case, we can rewrite (26) as

$$
G^{F}(h)=\max _{w}\left[h J_{\mathrm{st}}^{F+w} L-\int_{0}^{L} d x \frac{P_{\mathrm{st}}^{F+w}(x)}{4 T \gamma} w(x)^{2}\right],
$$

where $J_{\mathrm{st}}^{F+w}$ and $P_{\mathrm{st}}^{F+w}(x)$ are the stationary probability current and density in the modified system. Note that $J_{\mathrm{st}}^{F+w}$ and $P_{\mathrm{st}}^{F+w}(x)$ can be determined uniquely from (97) for a given extra force $w(x)$. Conversely, when a constant current $J$ and a probability density function $P(x)$ are given, we can define the extra force $w(x)$ by

$$
w(x)=\frac{\gamma}{P(x)}\left(J+\frac{T}{\gamma} \frac{\partial P(x)}{\partial x}-\frac{P(x)}{\gamma} F(x)\right) .
$$

Thus, there is a one-to-one correspondence between $w(x)$ and $\left(J_{\mathrm{st}}^{F+w}, P_{\mathrm{st}}^{F+w}(x)\right)$. From this fact, we can rewrite (122) as

$$
\begin{aligned}
& G^{F}(h)=\max _{P, J}[h J L \\
& \left.-\frac{1}{4 T} \int_{0}^{L} d x \frac{\gamma}{P(x)}\left(J+\frac{T}{\gamma} \frac{\partial P(x)}{\partial x}-\frac{P(x)}{\gamma} F(x)\right)^{2}\right] .
\end{aligned}
$$

By taking the maximum of $P(x)$ first, we obtain

$$
\begin{aligned}
& G^{F}(h)=\max _{J}[h J L \\
& \left.-\frac{1}{4 T} \min _{P}\left[\int_{0}^{L} d x \frac{\gamma}{P(x)}\left(J+\frac{T}{\gamma} \frac{\partial P(x)}{\partial x}-\frac{P(x)}{\gamma} F(x)\right)^{2}\right]\right] .
\end{aligned}
$$

Therefore, we find that the Legendre transform of $G^{F}(h)$ with respect to $h L$, which is the large deviation function $I(J)$, becomes

$I(J)$

$$
=\frac{1}{4 T} \min _{P}\left[\int_{0}^{L} d x \frac{\gamma}{P(x)}\left(J+\frac{T}{\gamma} \frac{\partial P(x)}{\partial x}-\frac{P(x)}{\gamma} F(x)\right)^{2}\right] .
$$

This expression is the same as the variational expression derived from the additivity principle [25 28].

When the system is described by fluctuating hydrodynamics, the variational expression is obtained under the assumption that the most dominant contribution to a path integration is given as a stationary configuration 29 33]. Here, since the Langevin equation (8) can be mapped to a stochastic partial differential equation [65], which is equivalent to fluctuating hydrodynamics, we can derive the variational expression in our system if the assumption is satisfied. Our result (126) indicates that the assumption is valid.

In the derivation of (126), the spatial homogeneity of the stationary current plays an important role. However, since this property is specific to one-dimensional systems, it is not obvious whether the same variational principle does hold in higher-dimensional cases [66]. Furthermore, by noticing that the probability current and the probability density considered here are equivalent to the particle current and the particle density in non-interacting particle systems, one might find that it is a highly non-trivial problem to derive (126) for interacting particle systems.

\section{Experimental determination of cumulant generating function}

We consider an experimental method for determining $G^{F}(h)$ without the information of $F(x)$. Since the direct evaluation of cumulants becomes harder and harder as the order of the cumulants increases, it may be useful if we could present an efficient method for evaluating $G^{F}(h)$ on the basis of our formula under the assumption that we measure only the trajectories $(x(t))_{t=0}^{\tau}$ and values of $T$ and $\gamma$.

The basic idea is to utilize (58), which was a cornerstone in the derivation of the main results (26) and (28). First, we add some external forces $w(x)$ to the system and measure trajectories of the particle. We then notice 
that the left-hand side of (58) is independent of external forces $w(x)$. Since we can estimate the right-hand side of (58) by using the measured data, we obtain equations which yield $w_{h}^{F, \text { opt }}(x)$. Essentially the same idea was reported in Ref. [35]. Below, we present a slightly different one from that in Ref. 35].

Concretely, in terms of a complete set of orthogonal functions $\left(\phi_{\alpha}\right)_{\alpha=0}^{\infty}$, we expand $w_{h}^{F, \text { opt }}(x)$ as

$$
w_{h}^{F, \text { opt }}(x) \simeq \sum_{\alpha=0}^{m} u_{\alpha}^{h} \phi_{\alpha}(x),
$$

where $m$ is a truncation number of the expansion. As $m$ is increased, the accuracy of the expansion (127) increases. For later convenience, we choose $\phi_{0}(x)=1$. Since $\int_{0}^{L} d x w_{h}^{F, \text { opt }}(x)=2 T h L$ (See (29) ), the coefficient $u_{0}^{h}$ is derived as

$$
u_{0}^{h}=2 T h
$$

We shall determine the other expansion coefficients $\left(u_{\alpha}^{h}\right)_{\alpha=1}^{m}$.

First, by differentiating (58) with respect to $h$, we obtain

$\frac{\partial G^{F}(h)}{\partial h}=\langle V\rangle_{\mathrm{st}}^{F+w}+\frac{1}{2 T \gamma}\left\langle\left(w_{h}^{F, \mathrm{opt}}-w\right) \frac{\partial w_{h}^{\mathrm{opt}}}{\partial h}\right\rangle_{\mathrm{st}}^{F+w}$

We then expand $w(x)$ as

$$
w(x)=\sum_{\alpha=0}^{m} w_{\alpha} \phi_{\alpha}(x) .
$$

By substituting (127) and (130) into (129), we rewrite (129) as

$$
\begin{aligned}
& \frac{\partial G^{F}(h)}{\partial h} \\
& =\langle V\rangle_{\mathrm{st}}^{F+w}+\frac{1}{2 T \gamma} \sum_{\alpha=0}^{m} \sum_{\eta=0}^{m}\left(u_{\alpha}^{h}-w_{\alpha}\right)\left\langle\phi_{\alpha} \phi_{\eta}\right\rangle_{\mathrm{st}}^{F+w} \frac{\partial u_{\eta}^{h}}{\partial h} .
\end{aligned}
$$

Here, by defining

$$
A_{\eta}^{w} \equiv \frac{1}{2 T \gamma} \sum_{\alpha=0}^{m}\left(u_{\alpha}^{h}-w_{\alpha}\right)\left\langle\phi_{\alpha} \phi_{\eta}\right\rangle_{\mathrm{st}}^{F+w},
$$

we rewrite (131) as

$$
\frac{\partial G^{F}(h)}{\partial h}=\langle V\rangle_{\mathrm{st}}^{F+w}+2 T A_{0}^{w}+\sum_{\eta=1}^{m} A_{\eta}^{w} \frac{\partial u_{\eta}^{h}}{\partial h}
$$

where we have used (128). We prepare $m+1$ external forces $w^{(0)}(x), w^{(1)}(x), \ldots, w^{(m)}(x)$. Recalling that (133) is valid for any external force $w(x)$, we equate the righthand side of (133) for the case $w^{(0)}(x)$ to the right-hand side of (133) for the other cases $w^{(\alpha)}(x)(\alpha=1, \ldots, m)$. The obtained equations are

$$
\begin{aligned}
& \langle V\rangle_{\mathrm{st}}^{F+w^{(0)}}+2 T A_{0}^{w^{(0)}}-\langle V\rangle_{\mathrm{st}}^{F+w^{(\alpha)}}-2 T A_{0}^{w^{(\alpha)}} \\
& =\sum_{\eta=1}^{m}\left(A_{\eta}^{w^{(\alpha)}}-A_{\eta}^{w^{(0)}}\right) \frac{\partial u_{\eta}^{h}}{\partial h}
\end{aligned}
$$

where $\alpha=1, \ldots, m$. We further define an $m \times m$ matrix as

$$
\bar{A}_{\alpha \eta} \equiv A_{\eta}^{w^{(\alpha)}}-A_{\eta}^{w^{(0)}}
$$

With the assumption that $\bar{A}$ is invertible, we derive

$$
\begin{gathered}
\frac{\partial u_{\eta}^{h}}{\partial h}=\sum_{\alpha=1}^{m}\left(\bar{A}^{-1}\right)_{\eta \alpha}\left[\langle V\rangle_{\mathrm{st}}^{F+w^{(0)}}+2 T A_{0}^{w^{(0)}}\right. \\
\left.-\langle V\rangle_{\mathrm{st}}^{F+w^{(\alpha)}}-2 T A_{0}^{w^{(\alpha)}}\right] .
\end{gathered}
$$

This expression implies that $\partial u_{\eta}^{h} / \partial h$ is expressed in terms of $\left(u_{\eta}^{h}\right)_{\eta=1}^{m},\langle V\rangle_{\mathrm{st}}^{F+w^{(\alpha)}}$ and $\left\langle\phi_{\eta} \phi_{\delta}\right\rangle_{\mathrm{st}}^{F+w^{(\alpha)}}$. Now, from trajectories of the particle in the modified systems with the external forces $w^{(\alpha)}(x)$ added, we approximately obtain $\langle V\rangle_{\mathrm{st}}^{F+w^{(\alpha)}}$ and $\left\langle\phi_{\eta} \phi_{\delta}\right\rangle_{\mathrm{st}}^{F+w^{(\alpha)}}$ in the form

$$
\langle V\rangle_{\mathrm{st}}^{F+w^{(\alpha)}} \simeq \frac{1}{\tau} \int_{0}^{\tau} d t \dot{x}(t)
$$

$$
\left\langle\phi_{\eta} \phi_{\delta}\right\rangle_{\mathrm{st}}^{F+w^{\alpha}} \simeq \frac{1}{\tau} \int_{0}^{\tau} d t \phi_{\eta}(x(t)) \phi_{\delta}(x(t)),
$$

with $0 \leq \eta, \delta \leq m$. Note that the approximation becomes exact in the limit $\tau \rightarrow \infty$. Therefore, we can express $\partial u_{\eta}^{h} / \partial h$ by $\left(u_{\eta}^{h}\right)_{\eta=1}^{m}$ from experimental data. By integrating $\partial u_{\eta}^{h} / \partial h$ with the condition $\left.u_{\eta}^{h}\right|_{h=0}=0$, $(1 \leq \eta \leq m)$, we obtain $\left(u_{\eta}^{h}\right)_{\eta=1}^{m}$ as a function of $h$. Similarly, (133) leads to the expression of $\partial G^{F}(h) / \partial h$ in terms of $\left(u_{\eta}^{h}\right)_{\eta=1}^{m}$. The integration yields $G^{F}(h)$, where we have used $\left.G^{F}(h)\right|_{h=0}=0$.

We performed a numerical experiment of the driven Brownian particle on a ring subject to a periodic potential $U(x)=U_{0} \cos (2 \pi x / L)$. We set $\phi_{k}(x)=\cos (2 \pi k x / L)$ $(0 \leq k \leq m / 2), \phi_{k}(x)=\sin (2 \pi x(k-m / 2) / L)(m / 2<$ $k \leq m)$. The $m+1$ external forces $\left(w_{k}(x)\right)_{k=0}^{m}$ are chosen as $\left(C \phi_{k}(x)\right)_{k=0}^{m}$, where $C$ is a constant. Following the recipe we described above, we obtain $G^{F}(h)$ and $w_{h}^{F, \text { opt }}(x)$ from trajectories of the particle. The results are displayed in Fig. 2. We also calculate $G^{F}(h)$ and $w_{h}^{F, \text { opt }}(x)$ by approximately evaluating the largest eigenvalue and the corresponding eigenfunction of the operator $\mathcal{L}_{h}^{(x) \dagger}$. See Appendix E for the calculation method. These two results are close to each other. It turns out that our experimental determination method is useful to obtain the accurate value of $G^{F}(h)$ from measured trajectories. 

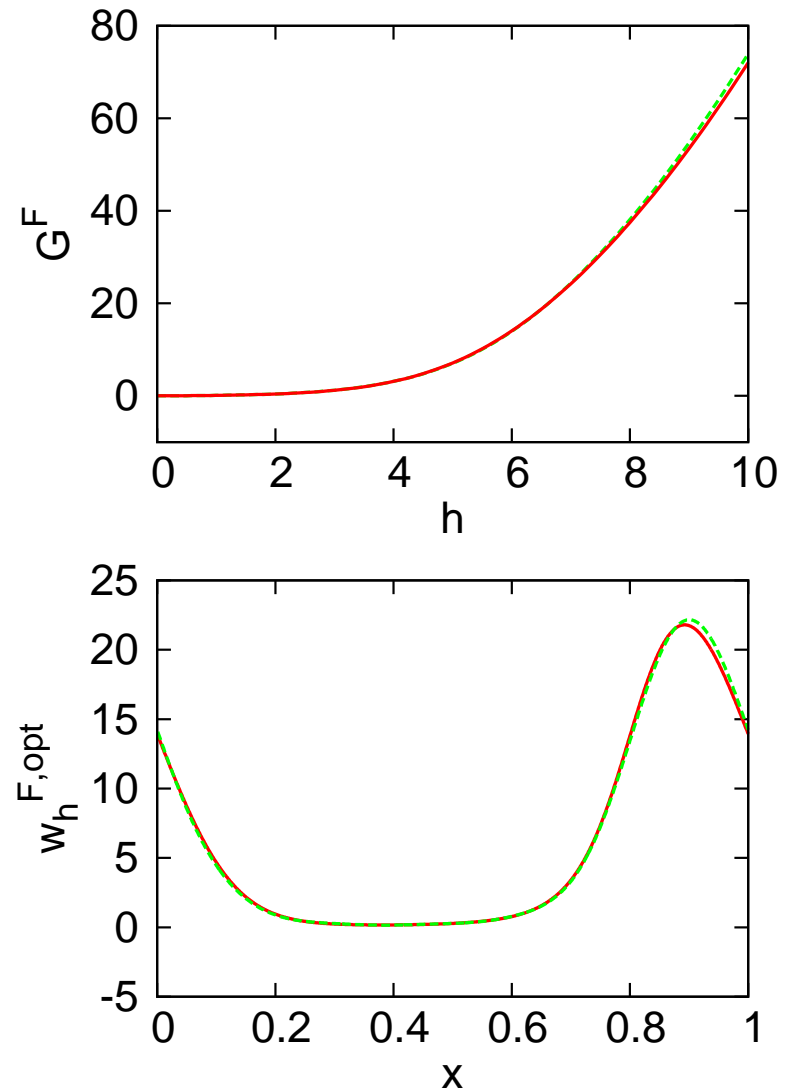

FIG. 2: (Color online) Numerical experiment for measurement of $G^{F}(h)$ (up) and $w_{h}^{F, \text { opt }}(x)$ with $h=3$ (down). Quantities are converted to dimensionless forms by setting $\gamma=T=L=1$. We fix $f=1$ and $U_{0}=3$. We assumed to know $T=\gamma=1$ and experimentally determined $G^{F}(h)$ and $w_{h}^{F, \text { opt }}(x)$ from trajectories $(x(t))_{t=0}^{\tau}$ following the method described in the text. We set $m=10, C=10$ and $\tau=400000$. By taking 10 samples, we estimated $G^{F}(h)$ and $w_{h}^{F, \text { opt }}(x)$. The obtained results are displayed with green dashed lines. Error-bars are within the lines. The red lines were obtained from the evaluation of the largest eigenvalue of $\mathcal{L}_{h}^{(x) \dagger}$.

\section{CONCLUDING REMARKS}

In this paper, we have presented the new formula (28) for the cumulant generating function of time-averaged velocity. The formula enables us to determine the cumulant generating function in terms of the expectation value of time-averaged current in a modified system. This corresponds to (A9) in the example of equilibrium statistical mechanics, and it is also regarded as an extension of the fluctuation-dissipation relation. Furthermore, we have pointed out that our formula is related to the additivity principle, the Donsker-Varadhan formula, and the least dissipation principle. Before ending the paper, we address problems that should be studied in future.

First, since our formulas are rather formal, we should explore further applications of our formulation to various physical problems. With regard to this aspect, in
Appendix E, we present a simple model in which we can explicitly calculate the cumulant generating function and the optimal force. We hope that this model might help readers to grasp our formulation and promote the applications. Furthermore, although our formulation is valid for interacting particle systems, useful predictions are not presented yet. A key step in the analysis of many-body problems is to extract slowly varying effective variables from many-body distribution functions. Here, we may employ some approximations for extracting effective variables. The result of the approximate calculation with our formulation might provide a new insight into properties of current fluctuations because our formulation is qualitatively new.

Next, we ask the range of the applicability of our formulation. The first question may be to consider similar formulas in under-damped Langevin systems. The formulas that correspond to (26) and (28) can be derived in a straight-forward manner. This fact is not surprising at all when the formulation of this paper is properly understood. However, it may be difficult to find a physical interpretation of the formulas. For example, it seems that "the extra force" appearing in the variational formula cannot be realized in laboratory experiments. More importantly, as far as we attempted, the variational function may not be expressed in terms of the entropy production rates, which is different from the over-damped case. We do not understand whether or not these are fatal in our formulation. We need further investigations.

Here, with regard to the range of the applicability of our formulas, we restrict our concerns to a formal aspect by putting aside physical interpretations. Concretely, in Markov jump processes, we can derive the formulas that correspond to (26) and (28). As seen in (G14) and (G15) in Appendix G] the mathematical expressions are quite simple. We thus expect that there might be physical examples for which these formulas are useful. Furthermore, since these are expressed in terms of escape rates, it is conjectured that similar variational principles are formulated in many-body Hamiltonian systems. It is stimulating to prove this conjecture. By combining physical interpretations and formal aspects, we wish to seek for a universal structure behind our formulation.

Finally, we go back to our basic motivation. Our naive idea is that the large deviation theory for current fluctuations might be associated with an operational framework. This was conjectured from the well-established fact that the large deviation theory for fluctuations of thermodynamic variables is tightly connected to equilibrium thermodynamics. The progress developed in this paper does not provide a final answer to the problem, but the results are encouraging us to continue our efforts. In the best case, we might have an operational framework of thermodynamics for currents. Then, we will consider a possibility to unify such a framework with equilibrium thermodynamics. It may be plausible that a unified scheme is related to the so-called steady state thermodynamics 19 21, 67 69]. With dreaming such possibilities, we chal- 
lenge to study operational viewpoints in non-equilibrium statistical mechanics.

\section{Acknowledgement}

The authors thank H. Tasaki for continuous discussions on non-equilibrium statistical mechanics. They also thank F. van Wijland for indicating the exactly calculable model in Appendix E. The present study was supported by grants from the Ministry of Education, Culture, Sports, Science, and Technology of Japan, Nos. 22340109 and 23654130.

\section{Appendix A: Equilibrium statistical mechanics}

We review the relation between the cumulant generating function of a thermodynamic variable and a thermodynamic function. Specifically, we consider a spin model with finite range interaction in a finite dimensional lattice $\Lambda$. Let $\sigma_{i}, i \in \Lambda$, be a spin variable whose value is either +1 or -1 . We denote $\left(\sigma_{i}\right)_{i \in \Lambda}$ by $\sigma$ collectively. We assume that the Hamiltonian for the system under a magnetic field $H$ is given by

$$
\mathcal{H}_{H}(\sigma)=\mathcal{H}_{0}(\sigma)-H \mu \sum_{i \in \Lambda} \sigma_{i}
$$

where $\mu$ is the magnetic moment. According to equilibrium statistical mechanics, for any observable $A(\sigma)$, the expectation value $\langle A\rangle_{T, H}$ in the system at temperature $T$ is given by

$$
\langle A\rangle_{T, H}=\sum_{\sigma} A(\sigma) \frac{e^{-\mathcal{H}_{H}(\sigma) / T}}{Z(T, H)},
$$

where

$$
Z(T, H)=\sum_{\sigma} e^{-\mathcal{H}_{H}(\sigma) / T}
$$

As an observable, we consider the magnetization density

$$
\mathrm{m}(\sigma)=\frac{\mu}{N} \sum_{i \in \Lambda} \sigma_{i}
$$

where $N=|\Lambda|$. Now, we define the free energy density $f(T, H)$ by

$$
f(T, H)=-T \lim _{N \rightarrow \infty} \frac{1}{N} \log Z(T, H) .
$$

Then, the fundamental relation of thermodynamics is written as

$$
d f=-s d T-m d H,
$$

where $s$ is the entropy density and $m$ is the expectation value of the magnetization density $\mathrm{m}(\sigma)$. Notice that
$f(T, H)$ is determined from the measurement of the heat capacity $c(T, H)$ and $m(T, H)$.

Putting aside the thermodynamic function, we consider the scaled cumulant generating function of $\mathrm{m}(\sigma)$, which is defined as

$$
G_{T, H}(h)=\lim _{N \rightarrow \infty} \frac{1}{N} \log \left\langle e^{h \mathrm{~m}(\sigma) N}\right\rangle_{T, H}
$$

From (A5) and (A7), we find the following relation between the cumulant generating function and the free energy:

$$
G_{T, H}(h)=-\frac{1}{T}[f(T, H+h T)-f(T, H)] .
$$

In particular, from the thermodynamic relation (A6), we obtain

$$
\frac{\partial G_{T, H}(h)}{\partial h}=m(T, H+h T)
$$

for any $h$. By differentiating (A9) with respect to $h$ and set $h=0$, we obtain

$$
\lim _{N \rightarrow \infty} N\left\langle(\mathrm{~m}(\sigma)-m)^{2}\right\rangle_{T, H}=T \frac{\partial m(T, H)}{\partial H}
$$

which is referred to as a fluctuation-response relation [1].

\section{Appendix B: Properties of the largest eigenvalue}

We show that the largest eigenvalue of the operators $\mathcal{L}_{\boldsymbol{h}}^{(\boldsymbol{x})}$ and $\mathcal{L}^{\dagger}{ }_{\boldsymbol{h}}^{(\boldsymbol{x})}$ is real and that the corresponding eigenfunctions are positive. The argument presented here is not mathematically rigorous, but provides a practical idea for understanding the claim. Although we focus on one-dimensional case for simplicity, the extension to higher-dimensional cases are straight-forward. We express the operator $\mathcal{L}_{h}^{(x)}$ in the form

$$
\mathcal{L}_{h}^{(x)} \cdot \varphi=A(x) \frac{\partial^{2}}{\partial x^{2}} \varphi+B(x) \frac{\partial}{\partial x} \varphi+C(x) \varphi,
$$

where $A(x)>0$. We study the eigenvalue problem of $\mathcal{L}_{h}^{(x)}$ in the space of periodic functions defined on the interval $[0, L]$.

We define $x_{i} \equiv L i / N, i=1,2, \ldots, N$, and let $\Delta x$ be the distance between $x_{i+1}-x_{i}$. We replace the differential operator $\partial / \partial x$ and $\partial^{2} / \partial x^{2}$ by differences. Concretely, the following replacements are considered:

$$
\begin{gathered}
\left.\frac{\partial \varphi}{\partial x}\right|_{x=x_{i}} \rightarrow \frac{\varphi\left(x_{i+1}\right)-\varphi\left(x_{i-1}\right)}{2 \Delta x}, \\
\left.\frac{\partial^{2} \varphi}{\partial x^{2}}\right|_{x=x_{i}} \rightarrow \frac{\varphi\left(x_{i+1}\right)+\varphi\left(x_{i-1}\right)-2 \varphi\left(x_{i}\right)}{(\Delta x)^{2}},
\end{gathered}
$$


for $i=2,3, \ldots, N-1$. A special care is needed for the cases $i=1$ and $i=N$. For example,

$$
\begin{gathered}
\left.\frac{\partial \varphi}{\partial x}\right|_{x=x_{1}} \rightarrow \frac{\varphi\left(x_{2}\right)-\varphi\left(x_{N}\right)}{2 \Delta x} \\
\left.\frac{\partial^{2} \varphi}{\partial x^{2}}\right|_{x=x_{N}} \rightarrow \frac{\varphi\left(x_{1}\right)+\varphi\left(x_{N-1}\right)-2 \varphi\left(x_{N}\right)}{(\Delta x)^{2}} .
\end{gathered}
$$

Then, we define an $N \times N$ matrix $M_{N}$ such that

$$
\begin{aligned}
& \left(M_{N} \varphi\right)_{i} \\
& =\left.A\left(x_{i}\right) \frac{\partial^{2} \varphi}{\partial x^{2}}\right|_{x=x_{i}}+\left.B\left(x_{i}\right) \frac{\partial \varphi}{\partial x}\right|_{x=x_{i}}+C\left(x_{i}\right) \varphi\left(x_{i}\right),
\end{aligned}
$$

where the $N$ dimensional vector $\varphi$ is defined by $(\varphi)_{i}=$ $\varphi\left(x_{i}\right)$. Since $M_{N}$ is an approximation of the operator $\mathcal{L}_{h}^{(x)}$, we assume that the eigenvalues and eigenfunctions of $\mathcal{L}_{h}^{(x)}$ are determined from the limit $N \rightarrow \infty$ for the eigenvalues and eigenvectors of $M_{N}$. Thus, below, we study the eigenvalue problem of $M_{N}$ instead of the eigenvalue problem of $\mathcal{L}_{h}^{(x)}$.

We show that the largest eigenvalue of $M_{N}$ is real and that all components of the corresponding left and right eigenvectors are positive, when $N$ is sufficiently large. This claim is understood from the Perron-Frobenius theory. A real matrix $B$ is called an irreducible ML-matrix [70] if $B$ satisfies the following two conditions: (i) All non-diagonal components of $B$ are non-negative. (ii) There exists a positive number $a$ such that the matrix $T \equiv a I+B$ is irreducible, where $I$ is the unit matrix. The Perron-Frobenius theory leads to the statement that the irreducible ML-matrix $B$ has an eigenvalue $\mu_{\mathrm{pf}}$ that satisfies the following conditions:

1. $\mu_{\mathrm{pf}}$ is real.

2. The associated left and right eigenvectors are strictly positive and unique up to constant multiples.

3. For any eigenvalue $\mu$ except for $\mu_{\mathrm{pf}}$, the real part of $\mu$ is less than $\mu_{\mathrm{pf}}$.

See Theorem 2.6 of Ref. 70]. Since $A(x)>0$, the matrix $M_{N}$ for sufficiently large $N$ turns out to be an irreducible ML-matrix. We thus obtain the claim that the largest eigenvalue of $M_{N}$ is real and that all components of the corresponding left and right eigenvectors are positive.

\section{Appendix C: $\sigma^{\boldsymbol{F}}(P)$ and entropy production rate}

We explain thermodynamic interpretations of the first and second terms of (70), which were denoted by $\Sigma^{\boldsymbol{F}}(P)$ and $\Omega^{\boldsymbol{F}}(P)$ in Section IV A, respectively.

We first consider the first term, $\Sigma^{\boldsymbol{F}}(P)$. We assume that the system is set under the force $\boldsymbol{F}$ and that the probability density is given by $P$. We define a probability density $p^{\boldsymbol{F}}(\boldsymbol{x}, t)$ as the solution of

$$
\frac{\partial}{\partial t} p^{\boldsymbol{F}}(\boldsymbol{x}, t)=-\sum_{i=1}^{N d} \frac{\partial}{\partial x_{i}} q_{i}^{\boldsymbol{F}}\left(\boldsymbol{x} \mid p^{\boldsymbol{F}}\right)
$$

with the initial condition

$$
p^{\boldsymbol{F}}(\boldsymbol{x}, 0)=P(\boldsymbol{x}) .
$$

Here, we take the limit $t \rightarrow 0$ in (C1). By noting (C2) in the right-hand side of (C1), we obtain

$$
\lim _{t \rightarrow 0} \frac{\partial}{\partial t} p^{\boldsymbol{F}}(\boldsymbol{x}, t)=-\sum_{i=1}^{N d} \frac{\partial}{\partial x_{i}} q_{i}^{\boldsymbol{F}}(\boldsymbol{x} \mid P) .
$$

Thus, the first term of (170),$\Sigma^{\boldsymbol{F}}(P)$ is expressed as

$$
\Sigma^{\boldsymbol{F}}(P)=-\lim _{t \rightarrow 0}\left\{\int_{\Omega^{N}} d \boldsymbol{x}\left[\log p^{\boldsymbol{F}}(\boldsymbol{x}, t)\right] \frac{\partial}{\partial t} p^{\boldsymbol{F}}(\boldsymbol{x}, t)\right\} .
$$

Since $\int_{\Omega^{N}} d \boldsymbol{x} p^{\boldsymbol{F}}(\boldsymbol{x}, t)=1$, (C4) is further rewritten as

$$
\Sigma^{\boldsymbol{F}}(P)=\left.\frac{d}{d t}\left(-\int_{\Omega^{N}} d \boldsymbol{x} p^{\boldsymbol{F}}(\boldsymbol{x}, t) \log p^{\boldsymbol{F}}(\boldsymbol{x}, t)\right)\right|_{t=0} .
$$

This shows that $\Sigma^{\boldsymbol{F}}(P)$ is the increasing rate of the Shannon entropy for a given distribution $P$.

Next, we consider the second term, $\Omega^{\boldsymbol{F}}(P)$. We shall show that $\Omega^{\boldsymbol{F}}(P)$ is the expectation value of the energy dissipation rate divided by $T$ when the distribution is given by $P$. According to the energetic interpretation of Langevin equations [7, 72], the energy dissipation rate $\Psi$ for each realization is given by

$$
\Psi=\sum_{i=1}^{N d}\left(\gamma_{i} \dot{x}_{i}-\sqrt{2 \gamma_{i} T} \xi_{i}\right) \circ \dot{x}_{i}
$$

where the symbol $\circ$ represents the multiplication in the sense of the Stratonovich [36]. It should be noted that $\Psi$ is a stochastic variable. By substituting (8) into (C6), we write

$$
\Psi=\sum_{i=1}^{N d}\left(\frac{1}{\gamma_{i}} F_{i}(\boldsymbol{x})^{2}+\sqrt{\frac{2 T}{\gamma_{i}}} F_{i}(\boldsymbol{x}) \circ \xi_{i}\right) .
$$

Here, we change the multiplication rule from the Stratonovich type (o) to the Ito type (.) [36]. Then, (C7) becomes

$$
\Psi=\sum_{i=1}^{N d}\left(\frac{1}{\gamma_{i}} F_{i}(\boldsymbol{x})^{2}+\frac{T}{\gamma_{i}} \frac{\partial F_{i}(\boldsymbol{x})}{\partial x_{i}}+\sqrt{\frac{2 T}{\gamma_{i}}} F_{i}(\boldsymbol{x}) \cdot \xi_{i}\right) .
$$

By taking the expectation value of (C8) and integrating by parts the second term, we obtain

$$
\begin{aligned}
\langle\Psi\rangle & =\int d \boldsymbol{x} \sum_{i=1}^{N d} F_{i}(\boldsymbol{x})\left(\frac{1}{\gamma_{i}} F_{i}(\boldsymbol{x}) P(\boldsymbol{x})-\frac{T}{\gamma_{i}} \frac{\partial P(\boldsymbol{x})}{\partial x_{i}}\right) \\
& =T \Omega^{\boldsymbol{F}}(P),
\end{aligned}
$$


where we have used $\left\langle F_{i}(\boldsymbol{x}) \cdot \xi_{i}\right\rangle=0$. The last expression claims that $\Omega^{\boldsymbol{F}}(P)$ is the expectation value of the energy dissipation rate divided by $T$ when the distribution is given by $P$.

\section{Appendix D: Iterative expression of cumulant coefficients}

For the system studied in Section $\nabla$, we determine the cumulant coefficients in an iterative manner. We start with the expression

$$
2 T G^{F}(h)=\frac{T}{\gamma} \frac{\partial u_{h}^{F}(x)}{\partial x}+\frac{u_{h}^{F}(x)}{\gamma}\left(F(x)+\frac{u_{h}^{F}(x)}{2}\right),
$$

which is obtained from (55) with $d=N=1$, where $u_{h}^{F}(x)$ satisfies

$$
\begin{gathered}
\int_{0}^{L} d x u_{h}^{F}(x)=2 T h L, \\
u_{h}^{F}(0)=u_{h}^{F}(L) .
\end{gathered}
$$

From a trivial relation

$$
\begin{aligned}
& \frac{\partial u_{h}^{F}(x)}{\partial x}+\frac{1}{T} u_{h}^{F}(x) F(x) \\
& =e^{-\int_{0}^{x} d y F(y) / T} \frac{\partial}{\partial x}\left[u_{h}^{F}(x) e^{\int_{0}^{x} d y F(y) / T}\right],
\end{aligned}
$$

we rewrite (D1) as

$$
\begin{aligned}
& \frac{\partial}{\partial x}\left[u_{h}^{F}(x) e^{\int_{0}^{x} d y F(y) / T}\right] \\
& =e^{\int_{0}^{x} d y F(y) / T}\left(2 \gamma G^{F}(h)-\frac{1}{2 T} u_{h}^{F}(x)^{2}\right) .
\end{aligned}
$$

By integrating (D5) with respect to $x$, we obtain

$$
\begin{aligned}
& u_{h}^{F}(x)=e^{-\int_{0}^{x} d y F(y) / T} \\
& \times\left[C+\int_{0}^{x} d y e^{\int_{0}^{y} d z F(z) / T}\left(2 \gamma G^{F}(h)-\frac{1}{2 T} u_{h}^{F}(y)^{2}\right)\right],
\end{aligned}
$$

where $C$ is an integration constant. Here, by direct calculation, we can derive an identity

$$
\begin{aligned}
& \int_{0}^{x} d y g(y) e^{\int_{0}^{y} d z F(z) / T}=\frac{1}{1-e^{f L / T}} \\
& {\left[\int_{0}^{L} d y g(y) e^{\int_{0}^{y} d z F(z) / T}-\int_{0}^{L} d y g(x+y) e^{\int_{0}^{x+y} d z F(z) / T}\right]}
\end{aligned}
$$

for any periodic function $g(x)$. We then set

$$
g(x)=2 \gamma G^{F}(h)-\frac{1}{2 T} u_{h}^{F}(x)^{2}
$$

in (D7) and substitute it into the second term of the right-hand side of (D6). The result is

$$
\begin{aligned}
& u_{h}^{F}(x)=e^{-\int_{0}^{x} d y F(y) / T}\left[\tilde{C}-\frac{1}{1-e^{f L / T}}\right. \\
& \left.\times \int_{0}^{L} d y e^{\int_{0}^{x+y}} d z F(z) / T\left(2 \gamma G^{F}(h)-\frac{1}{2 T} u_{h}^{F}(x+y)^{2}\right)\right],
\end{aligned}
$$

where $\tilde{C}$ is a constant. The condition $u_{h}^{F}(0)=u_{h}^{F}(L)$ leads to $\tilde{C}=0$. Through the transformation of the integration variable $y \rightarrow L-y$, we rewrite (D9) as

$$
\begin{aligned}
& u_{h}^{F}(x)=\frac{2 \gamma}{1-e^{-f L / T}} \\
& {\left[G^{F}(h) I_{+}(x)-\frac{1}{4 T \gamma} \int_{0}^{L} d y e^{\int_{x}^{x-y} d z F(z) / T} u_{h}^{F}(x-y)^{2}\right],}
\end{aligned}
$$

where we have used (98). By integrating the both sides of (D10) with respect to $x$ and using the condition (D2), we derive

$$
G^{F}(h)=h L \frac{T}{\gamma}\left(1-e^{-f L / T}\right) \alpha+\frac{\alpha}{4 T \gamma} \int_{0}^{L} d x I_{-}(x) u_{h}^{F}(x)^{2},
$$

where $\alpha$ was defined in (102).

It should be noted that $u_{h}^{F}(x)$ satisfies a self-consistent equation. Indeed, by substituting (D11) into (D10), we find

$$
\begin{aligned}
& u_{h}^{F}(x)=2 T h L \alpha I_{+}(x)+\frac{1}{2 T\left(1-e^{-f L / T}\right)} \int_{0}^{L} d y \\
& {\left[e^{\int_{x}^{x-y} d z F(z) / T}\left(\alpha \int_{0}^{L} d r I_{-}(r) u_{h}^{F}(r)^{2}-u_{h}^{F}(x-y)^{2}\right)\right] .}
\end{aligned}
$$

By substituting (105) into (D12), we can determine the coefficient $u_{n}(x)$ iteratively. Concretely, by noting

$$
\begin{aligned}
u_{h}^{F}(x)^{2} & =\sum_{n=1}^{\infty} \sum_{l=1}^{\infty} u_{n}(x) u_{l}(x) h^{n+l} \\
& =\sum_{n=2}^{\infty} h^{n}\left(\sum_{l=1}^{n-1} u_{l}(x) u_{n-l}(x)\right)
\end{aligned}
$$

we derive a recursion equation

$$
\begin{aligned}
& u_{n}(x)=\frac{1}{2 T\left(1-e^{-f L / T}\right)} \sum_{l=1}^{n-1} \int_{0}^{L} d y e^{\int_{x}^{x-y} d z F(z) / T} \\
& {\left[\alpha\left(\int_{0}^{L} d r I_{-}(r) u_{l}(r) u_{n-l}(r)\right)-u_{l}(x-y) u_{n-l}(x-y)\right]}
\end{aligned}
$$


for $n \geq 2$. For example, by recalling (114), we calculate

$$
\begin{aligned}
u_{2}(x)= & \frac{2 T \alpha^{2} L^{2}}{\left(1-e^{-f L / T}\right)} \int_{0}^{L} d y e^{\int_{x}^{x-y} d z F(z) / T} \\
& {\left[\alpha\left(\int_{0}^{L} d r I_{-}(r) I_{+}(r)^{2}\right)-I_{+}(x-y)^{2}\right] . }
\end{aligned}
$$

Furthermore, we expand $G^{F}(h)$ as

$$
G^{F}(h)=\sum_{n=1}^{\infty} \frac{G_{n}}{n !} h^{n} .
$$

By substituting (D13) and (D16) into (D11), we obtain

$$
G_{1}=L \frac{T}{\gamma}\left(1-e^{-f L / T}\right) \alpha
$$

and

$$
G_{n}=\sum_{l=1}^{n-1} \frac{\alpha n !}{4 T \gamma} \int_{0}^{L} d x I_{-}(x) u_{l}(x) u_{n-l}(x)
$$

for $n \geq 2$. With this formula, the cumulant coefficients $G_{n}$ can be calculated in an iterative manner. For example, the third-order cumulant coefficient is derived as

$$
G_{3}=\frac{6 \alpha^{2} L}{\gamma} \int_{0}^{L} d x I_{-}(x) I_{+}(x) u_{2}(x) .
$$

Reimann et al found the exact expression of the diffusion constant (100) from the first and second cumulants of first passage time [39]. Here, we remember an iterative formula for the moments of the first passage time [36]. By comparing this iterative formula with our iterative formula, one might find a general relationship between the cumulant generating function and statistical properties of the first passage time beyond the first two cumulants.

\section{Appendix E: Calculation of $G(h)$ and $w_{h}^{\mathrm{opt}}(x)$}

We focus on the case that $N=1$ and $d=1$, which is studied in section $\mathrm{V}$. We numerically solve the largest eigenvalue problem (51). First, we consider a linear equation

$$
\frac{\partial}{\partial t} \phi(x, t)=\mathcal{L}_{h}^{(x) \dagger} \phi(x, t) .
$$

Since $G^{F}(h)$ is the largest eigenvalue of $\mathcal{L}_{h}^{(x) \dagger}$, the long time behaviors of solutions are described as

$$
\phi(x, t) \simeq \psi_{h}(x) e^{G^{F}(h) t} .
$$

Now, we assume $\phi(x, t)>0$ for any $t$ by choosing appropriate initial conditions. Then, we define

$$
u(x, t) \equiv 2 T \frac{\partial}{\partial x} \log \phi(x, t)+2 T h .
$$

By dividing (E1) by $\phi(x, t)$ and using the same method deriving (55) in section III.A, we obtain

$2 T \frac{\partial}{\partial t} \log \phi(x, t)=\frac{F(x)}{\gamma} u(x, t)+\frac{1}{2 \gamma} u(x, t)^{2}+\frac{T}{\gamma} \frac{\partial}{\partial x} u(x, t)$.

Finally, by differentiating (E4) with respect to $x$, we rewrite (E4) as

$$
\frac{\partial}{\partial t} u(x, t)=-\frac{\partial}{\partial x} j_{u}(x, t),
$$

with a current

$$
j_{u}(x, t) \equiv-\frac{F(x)}{\gamma} u(x, t)-\frac{1}{2 \gamma} u(x, t)^{2}-\frac{T}{\gamma} \frac{\partial}{\partial x} u(x, t) .
$$

Let $u_{*}(x)$ be the stationary solution given as the longtime limit of $u(x, t)$. Then, from (E2) and (E3), we obtain

$$
u_{*}(x)=2 T h \frac{\partial}{\partial x} \log \psi_{h}(x)+2 T h .
$$

By recalling (29), we have

$$
w_{h}^{\mathrm{opt}}(x)=u_{*}(x) .
$$

Furthermore, by comparing (E6) with (55), we obtain

$$
G^{F}(h)=-\frac{1}{2 T} \lim _{t \rightarrow \infty} j_{u}(x, t) .
$$

Therefore, by numerically solving (E5), we can calculate $G^{F}(h)$ and $w_{h}^{\mathrm{opt}}(x)$.

\section{Appendix F: Exactly calculable model}

We illustrate our theoretical idea by explicitly deriving the cumulant generating function and the optimal force for a linear Langevin equation. The model describes the motion of a single Brownian particle in two dimensions, which corresponds to the case that $N=1$ and $d=2$ in (8). By setting $\gamma=1$ for notational simplicity, we write the equation as

$$
\frac{d \boldsymbol{x}}{d t}=\boldsymbol{F}(\boldsymbol{x})+\sqrt{2 T} \boldsymbol{\xi}
$$

with

$$
\boldsymbol{F}(\boldsymbol{x}) \equiv-k \boldsymbol{x}+\epsilon \boldsymbol{l}(\boldsymbol{x}),
$$

where $\boldsymbol{l}=\left(-x_{2}, x_{1}\right)$. From (11) and (12), we obtain the stationary distribution as

$$
P_{\mathrm{st}}^{\boldsymbol{F}}(\boldsymbol{x})=C \exp \left(-\frac{k}{2 T} \boldsymbol{x}^{2}\right),
$$

with a normalization constant $C$. Although the stationary distribution is Gaussian, the detailed balance condition is not satisfied because of the circulation force $\epsilon \boldsymbol{l}$. 
One can check this fact immediately from (11) and (F3). It should be noted that the linear Langevin equation was studied with a novel idea, irreversible circulation, which characterizes the violation of detailed balance [73].

The quantity we consider here is a time-averaged angular momentum (or irreversible circulation) defined as

$$
L(\tau) \equiv \frac{1}{\tau} \int_{0}^{\tau} d t \boldsymbol{l}(\boldsymbol{x}(t)) \cdot \dot{\boldsymbol{x}}(t) .
$$

For the scaled cumulant generating function of $L$,

$$
G(h)=\lim _{\tau \rightarrow \infty} \frac{1}{\tau} \log \left\langle e^{\tau h L(\tau)}\right\rangle,
$$

we can derive the variational principle by using the same method as Section III A. First, we define an operator $\mathcal{L}_{h}$ as

$$
\begin{aligned}
\mathcal{L}_{h} \cdot \varphi & =T \nabla^{2} \varphi-\nabla \cdot(\boldsymbol{F} \varphi)+h(\boldsymbol{l} \cdot \boldsymbol{F}) \varphi \\
& +h^{2} T \boldsymbol{l}^{2} \varphi-2 T h \boldsymbol{\nabla} \cdot(\boldsymbol{l} \varphi)
\end{aligned}
$$

Since $G(h)$ is equal to the largest eigenvalue of the operators $\mathcal{L}_{h}$ and $\mathcal{L}_{h}^{\dagger}$, we have

$$
\mathcal{L}_{h}^{\dagger} \cdot \phi=G(h) \phi
$$

where $\phi$ is the positive eigenfunction corresponding to the largest eigenvalue of $\mathcal{L}_{h}^{\dagger}$. Next, we define a force $\boldsymbol{u}$ as

$$
\boldsymbol{u}(\boldsymbol{x})=2 T(\boldsymbol{\nabla} \log \phi(\boldsymbol{x})+h \boldsymbol{l}(\boldsymbol{x})) .
$$

By dividing ( $(\mathrm{F} 7)$ by $\phi$ and using the definition of $\boldsymbol{u}$, we obtain

$$
\boldsymbol{F} \cdot \boldsymbol{u}+\frac{1}{2} \boldsymbol{u}^{2}+T \nabla \cdot \boldsymbol{u}=2 T G(h) .
$$

This takes the same form as (55) so that we can follow the argument from (55) to (58). We then arrive at

$$
G(h)=\tilde{\Phi}_{h}(\boldsymbol{w})+\frac{1}{4 T}\left\langle(\boldsymbol{w}-\boldsymbol{u})^{2}\right\rangle_{\mathrm{st}}^{\boldsymbol{F}+\boldsymbol{w}},
$$

with

$$
\tilde{\Phi}_{h}(\boldsymbol{w})=h \int_{\Omega} d \boldsymbol{x} \boldsymbol{l} \cdot \boldsymbol{j}_{\mathrm{st}}^{\boldsymbol{F}+\boldsymbol{w}}-\frac{1}{4 T}\left\langle\boldsymbol{w}^{2}\right\rangle_{\mathrm{st}}^{\boldsymbol{F}+\boldsymbol{w}}
$$

Here, it should be noted that

$$
\int_{\Omega} d \boldsymbol{x} \boldsymbol{l} \cdot \boldsymbol{j}_{\mathrm{st}}^{\boldsymbol{F}+\boldsymbol{w}}=\langle L\rangle_{\mathrm{st}}^{\boldsymbol{F}+\boldsymbol{w}} .
$$

(F10) is the key identity of our formulation, which corresponds to (58). Indeed, from (F10) we obtain

$$
\begin{gathered}
G(h)=\max _{\boldsymbol{w}} \Phi_{h}(\boldsymbol{w}), \\
\frac{\partial G(h)}{\partial h}=\langle L\rangle_{\mathrm{st}}^{\boldsymbol{F}+\boldsymbol{w}_{\mathrm{opt}}},
\end{gathered}
$$

where the optimal force $\boldsymbol{w}_{\mathrm{opt}}$ is given as

$$
\boldsymbol{w}_{\mathrm{opt}}(\boldsymbol{x})=\boldsymbol{u}(\boldsymbol{x})
$$

Finally, by solving (F9), we derive the explicit expression of $\boldsymbol{u}$ and $G(h)$. Suppose that

$$
\phi=\exp \left(-\frac{a}{2} x^{2}\right)
$$

where $a$ is a constant. This yields

$$
\boldsymbol{u}(\boldsymbol{x})=-2 T a \boldsymbol{x}+2 \operatorname{Th} \boldsymbol{l}(\boldsymbol{x}) .
$$

By substituting (F17) into (F9), we obtain

$$
G(h)=-2 T a+\boldsymbol{x}^{2}\left[T a^{2}+k a+h(T h+\epsilon)\right] .
$$

Since the left-hand side is independent of $\boldsymbol{x}$, we determine the constant $a$ as

$$
a=\frac{1}{2 T}\left(-k+\sqrt{k^{2}-4 T h(T h+\epsilon)}\right) .
$$

Here, we can confirm that (F7) holds for (F16) with (F19), because (F7) is equivalent to (F9). We then obtain

$$
G(h)=k-\sqrt{k^{2}-4 T h(T h+\epsilon)} .
$$

From (F17) and ( $(\mathrm{F19})$, the force in the optimal system is explicitly written as

$$
\boldsymbol{F}(\boldsymbol{x})+\boldsymbol{u}(\boldsymbol{x})=-\sqrt{k^{2}-4 T h(T h+\epsilon)} \boldsymbol{x}+(\epsilon+2 T h) \boldsymbol{l}(\boldsymbol{x}) .
$$

\section{Appendix G: Formula in Markov jump process}

The model we consider here is a continuous-time Markov process of a finite space $\mathcal{M}$. We assume that the transition rate, $w\left(\boldsymbol{a} \rightarrow \boldsymbol{a}^{\prime}\right)$ for $\boldsymbol{a} \neq \boldsymbol{a}^{\prime} \in \mathcal{M}$, is irreducible. We set $w(\boldsymbol{a} \rightarrow \boldsymbol{a})=0$ for notational simplicity. The escape rate $\lambda(\boldsymbol{a})$ is then defined by

$$
\lambda(\boldsymbol{a})=\sum_{\boldsymbol{a}^{\prime} \in \mathcal{M}} w\left(\boldsymbol{a} \rightarrow \boldsymbol{a}^{\prime}\right) .
$$

Let $P^{w}(\boldsymbol{a}, t)$ be a probability of $\boldsymbol{a}(t)=\boldsymbol{a}$, where the superscript $w$ represents the transition rate dependence of the probability $P^{w}$. Then, $P^{w}(\boldsymbol{a}, t)$ satisfies the master equation

$$
\frac{\partial}{\partial t} P^{w}(\boldsymbol{a}, t)=\sum_{\boldsymbol{a}^{\prime}}\left[w\left(\boldsymbol{a}^{\prime} \rightarrow \boldsymbol{a}\right)-\lambda(\boldsymbol{a}) \delta_{\boldsymbol{a}, \boldsymbol{a}^{\prime}}\right] P^{w}\left(\boldsymbol{a}^{\prime}, t\right)
$$

For later convenience, we define a matrix by

$$
\mathcal{A}\left(\boldsymbol{a}, \boldsymbol{a}^{\prime}\right) \equiv w\left(\boldsymbol{a}^{\prime} \rightarrow \boldsymbol{a}\right)-\lambda(\boldsymbol{a}) \delta_{\boldsymbol{a}, \boldsymbol{a}^{\prime}}
$$

The stationary distribution $P_{\mathrm{st}}^{w}(\boldsymbol{a})$ satisfies

$$
\sum_{\boldsymbol{a}^{\prime}} \mathcal{A}\left(\boldsymbol{a}, \boldsymbol{a}^{\prime}\right) P_{\mathrm{st}}^{w}\left(\boldsymbol{a}^{\prime}\right)=0 .
$$


We denote by $\langle g\rangle_{\text {st }}^{w}$ the expectation value of a function $g(\boldsymbol{a})$ with respect to the stationary distribution $P_{\mathrm{st}}^{w}(\boldsymbol{a})$.

We fix a time interval $[0, \tau]$. For a given history $(\boldsymbol{a}(t))_{t=0}^{\tau}$, where $\boldsymbol{a}(t) \in \mathcal{M}$, a sequence of transition times $\left(t_{1}, t_{2}, \cdots, t_{n-1}\right)$ is determined, and $\boldsymbol{a}(t)$ is expressed as $\boldsymbol{a}(t)=\boldsymbol{a}_{i}$ for $t_{i-1}<t<t_{i}$ with $t_{0}=0$ and $t_{n}=\tau$. Here, we consider a quantity $\alpha\left(\boldsymbol{a}_{i}, \boldsymbol{a}_{i+1}\right)$ defined for the jump event $\boldsymbol{a}_{i} \rightarrow \boldsymbol{a}_{i+1}$ at $t=t_{i}(1 \leq i \leq n-1)$. Its time-averaged value in the history is written as

$$
A(\tau)=\frac{1}{\tau} \sum_{i=1}^{n-1} \alpha\left(\boldsymbol{a}_{i}, \boldsymbol{a}_{i+1}\right) .
$$

We then study the scaled cumulant generating function of $A(\tau)$, which is defined by

$$
G(h) \equiv \lim _{\tau \rightarrow \infty} \frac{1}{\tau} \log \left\langle e^{\tau h A(\tau)}\right\rangle,
$$

where \langle\rangle represents the sample average over realizations of history with an initial distribution.

It is known that $G(h)$ is equal to the largest eigenvalue of the matrix $\mathcal{A}_{h}$ given by

$$
\mathcal{A}_{h}\left(\boldsymbol{a}^{\prime}, \boldsymbol{a}\right)=w\left(\boldsymbol{a} \rightarrow \boldsymbol{a}^{\prime}\right) e^{h \alpha\left(\boldsymbol{a}, \boldsymbol{a}^{\prime}\right)}-\lambda(\boldsymbol{a}) \delta_{\boldsymbol{a}, \boldsymbol{a}^{\prime}} .
$$

See Ref. 54] for the derivation. Let $\phi_{\max }(\boldsymbol{a})$ be the left eigenvector for the largest eigenvalue of $\mathcal{A}_{h}$. That is,

$$
\sum_{\boldsymbol{a}^{\prime}} \phi_{\max }\left(\boldsymbol{a}^{\prime}\right) \mathcal{A}_{h}\left(\boldsymbol{a}^{\prime}, \boldsymbol{a}\right)=G(h) \phi_{\max }(\boldsymbol{a}) .
$$

Since $\phi_{\max }(\boldsymbol{a})>0$ (See Appendix B), we may define a potential function $V_{\max }(\boldsymbol{a})$ as

$$
V_{\max }(\boldsymbol{a}) \equiv-2 \log \phi_{\max }(\boldsymbol{a}) .
$$

Dividing the both sides of (G8) by $\phi_{\max }(\boldsymbol{a})$, we obtain

$$
\begin{aligned}
& G(h) \\
& =\sum_{\boldsymbol{a}^{\prime}}\left[w\left(\boldsymbol{a} \rightarrow \boldsymbol{a}^{\prime}\right) e^{-V_{\max }\left(\boldsymbol{a}^{\prime}\right) / 2+V_{\max }(\boldsymbol{a}) / 2+h \alpha\left(\boldsymbol{a}, \boldsymbol{a}^{\prime}\right)}\right. \\
& \left.\quad-w\left(\boldsymbol{a} \rightarrow \boldsymbol{a}^{\prime}\right)\right] .
\end{aligned}
$$

Here, for any potential function $V(\boldsymbol{a})$, we define a modified transition rate $u_{h}^{V}\left(\boldsymbol{a} \rightarrow \boldsymbol{a}^{\prime}\right)$ as

$$
\begin{aligned}
& u_{h}^{V}\left(\boldsymbol{a} \rightarrow \boldsymbol{a}^{\prime}\right) \\
& \equiv w\left(\boldsymbol{a} \rightarrow \boldsymbol{a}^{\prime}\right) e^{-V\left(\boldsymbol{a}^{\prime}\right) / 2+V(\boldsymbol{a}) / 2+h \alpha\left(\boldsymbol{a}, \boldsymbol{a}^{\prime}\right)} .
\end{aligned}
$$

The corresponding escape rate $\lambda_{h}^{V}$ becomes

$$
\lambda_{h}^{V}(\boldsymbol{a})=\sum_{\boldsymbol{a}^{\prime}} u_{h}^{V}\left(\boldsymbol{a} \rightarrow \boldsymbol{a}^{\prime}\right),
$$

and (G10) is rewritten as

$$
G(h)=\lambda_{h}^{V_{\max }}(\boldsymbol{a})-\lambda(\boldsymbol{a}) .
$$

This expression might be known, although we do not find its explicit presentation in previous studies.

Now, our main claim, which is far from trivial, is the variational expression

$$
G(h)=\max _{V}\left\langle\lambda_{h}^{V}-\lambda\right\rangle_{\mathrm{st}}^{u_{h}^{V}},
$$

and the relation

$$
\frac{\partial G(h)}{\partial h}=\sum_{\boldsymbol{a}, \boldsymbol{a}^{\prime}} P_{\mathrm{st}}^{u_{\mathrm{opt}}^{V_{\mathrm{opt}}}}(\boldsymbol{a}) u_{h}^{V_{\mathrm{opt}}}\left(\boldsymbol{a} \rightarrow \boldsymbol{a}^{\prime}\right) \alpha\left(\boldsymbol{a}, \boldsymbol{a}^{\prime}\right)
$$

with

$$
V_{\mathrm{opt}}=\underset{V}{\operatorname{argmax}}\left\langle\lambda_{h}^{V}-\lambda\right\rangle_{\mathrm{st}}^{u_{h}^{V}}
$$

The derivation is the following. First, we consider the variation $V \rightarrow V+\delta V$ in $\left\langle\lambda_{h}^{V}-\lambda\right\rangle_{\text {st }}^{u_{h}^{V}}$, which is expressed as

$$
\begin{aligned}
& \delta\left[\left\langle\lambda_{h}^{V}-\lambda\right\rangle_{\mathrm{st}}^{u_{h}^{V}}\right] \\
& =\sum_{\boldsymbol{a}} \delta P_{\mathrm{st}}^{u_{h}^{V}}(\boldsymbol{a})\left[\lambda_{h}^{V}(\boldsymbol{a})-\lambda(\boldsymbol{a})\right]+\sum_{\boldsymbol{a}} P_{\mathrm{st}}^{u_{h}^{V}}(\boldsymbol{a}) \delta \lambda_{h}^{V}(\boldsymbol{a}) .
\end{aligned}
$$

From (G11) and (G12), we rewrite the second term of (G17) as

$$
-\frac{1}{2} \sum_{\boldsymbol{a}} \sum_{\boldsymbol{a}^{\prime}} P_{\mathrm{st}}^{u_{h}^{V}}(\boldsymbol{a}) u_{h}^{V}\left(\boldsymbol{a} \rightarrow \boldsymbol{a}^{\prime}\right)\left[\delta V\left(\boldsymbol{a}^{\prime}\right)-\delta V(\boldsymbol{a})\right] .
$$

Since this becomes zero from the condition of the stationary distribution, the variational equation $\delta\left[\left\langle\lambda_{h}^{V}-\lambda\right\rangle_{\mathrm{st}}^{u_{h}^{V}}\right]=0$ leads to

$$
K=\lambda_{h}^{V}(\boldsymbol{a})-\lambda(\boldsymbol{a}),
$$

where $K$ is a constant corresponding to a Lagrange multiplier. Here, G19 is equivalent to

$$
\sum_{\boldsymbol{a}^{\prime}} e^{-V_{\mathrm{opt}}\left(\boldsymbol{a}^{\prime}\right) / 2} \mathcal{A}_{h}\left(\boldsymbol{a}^{\prime}, \boldsymbol{a}\right)=K e^{-V_{\mathrm{opt}}(\boldsymbol{a}) / 2} .
$$

By comparing this with (G8), we find that $V_{\mathrm{opt}}=V_{\max }$ as the result of the Perron-Frobenius theory.

Next, we consider the modified system with the transition rate $u_{h}^{V_{\mathrm{opt}}}\left(\boldsymbol{a} \rightarrow \boldsymbol{a}^{\prime}\right)$. By multiplying the both sides of (G13) by $P_{\mathrm{st}}^{u_{h}^{V_{\mathrm{opt}}}}(\boldsymbol{a})$ and summing over $\boldsymbol{a}$, we obtain

$$
G(h)=\left\langle\lambda_{h}^{V_{\mathrm{opt}}}-\lambda\right\rangle_{\mathrm{st}}^{u_{h}^{V_{\mathrm{opt}}}},
$$

where we have used $V_{\text {opt }}=V_{\max }$. We thus conclude (G14). Furthermore, by differentiating (G13) with respect to $h$, multiplying the both sides by $P_{\mathrm{st}}^{u_{h}^{V_{\mathrm{opt}}}}(\boldsymbol{a})$, and 
summing over $\boldsymbol{a}$, we derive

$\frac{\partial G(h)}{\partial h}=\sum_{\boldsymbol{a}, \boldsymbol{a}^{\prime}} P_{\mathrm{st}}^{u_{h}^{V_{\mathrm{opt}}}}(\boldsymbol{a}) u_{h}^{V_{\mathrm{opt}}}\left(\boldsymbol{a} \rightarrow \boldsymbol{a}^{\prime}\right) \alpha\left(\boldsymbol{a}, \boldsymbol{a}^{\prime}\right)$
$-\frac{1}{2} \sum_{\boldsymbol{a}, \boldsymbol{a}^{\prime}} P_{\mathrm{st}}^{u_{h}^{V_{\mathrm{opt}}}}(\boldsymbol{a}) u_{h}^{V_{\mathrm{opt}}}\left(\boldsymbol{a} \rightarrow \boldsymbol{a}^{\prime}\right)\left(\frac{\partial V_{\mathrm{opt}}\left(\boldsymbol{a}^{\prime}\right)}{\partial h}-\frac{\partial V_{\mathrm{opt}}(\boldsymbol{a})}{\partial h}\right)$.

(G22)

The second term of (G22) turns out to be zero from the condition of the stationary distribution. The final expression is (G15).

At the end of this Appendix, we point out that the expression (G14) is similar to the formula of the large deviation function of the empirical measure in Markov jump processes [55]. This similarity corresponds to the relation between (72) and (82) in Langevin equations.
[1] C. H. Callen, Thermodynamics and an Introduction to Thermostatistics, 2nd Edition (Wiley, New York, 1985).

[2] A. Dembo and O. Zeitouni, Large deviations techniques and applications (Springer, New York, 1998).

[3] R. Kubo, M. Toda, and N. Hashitsume, Statistical Physics II: Nonequilibrium Statistical Mechanics (Springer, Berlin, 1991).

[4] R. Zwanzig, Nonequilibrium statistical mechanics (Oxford, New York, 2001).

[5] L. Onsager, Phys. Rev. 37, 405 (1931).

[6] L. Onsager, Phys. Rev. 38, 2265 (1931).

[7] L. Onsager and S. Machlup, Phys. Rev. 91, 1505 (1953).

[8] Y. Oono, Prog. Theor. Phys. 89, 973 (1993).

[9] D. J. Evans, E. G. D. Cohen, and G. P. Morriss, Phys. Rev. Lett. 71, 2401 (1993).

[10] G. Gallavotti and E. G. D. Cohen, Phys. Rev. Lett. 74, 2694 (1995).

[11] J. Kurchan, J. Phys. A 31, 3719 (1998).

[12] C. Maes, J. Stat. Phys. 95, 367 (1999).

[13] G. E. Crooks, Phys. Rev. E 60, 2721 (1999).

[14] J. L. Lebowitz and H. Spohn, J. Stat. Phys. 95, 333 (1999).

[15] K. Kawasaki and J. D. Gunton, Phys. Rev. A 8, 2048 (1973).

[16] D. N. Zubarev, Nonequlibrium Statistical Thermodynamics (Consultants Bureau, New York, 1974).

[17] J. A. McLennan, Introduction to Nonequilibrium Statistical Mechanics (Prentice-Hall, Englewood Cliffs, NJ, 1989).

[18] K. Hayashi and S.-i. Sasa, Physica A 370, 407 (2006) .

[19] T. S. Komatsu, N. Nakagawa, S.-i. Sasa, and H. Tasaki, Phys. Rev. Lett. 100, 230602 (2008).

[20] T. S. Komatsu, N. Nakagawa, S.-i. Sasa, and H. Tasaki, J. Stat. Phys. 142, 127 (2011).

[21] K. Saito and H. Tasaki, arXiv:1105.2168

[22] T. S. Komatsu and N. Nakagawa, Phys. Rev. Lett. 100, 030601 (2008).

[23] B. Derrida and J. L. Lebowitz, Phys. Rev. Lett. 80, 209 (1998).

[24] B. Derrida, Phys. Rep. 301, 65 (1998).

[25] T. Bodineau and B. Derrida, Phys. Rev. Lett. 92, 180601 (2004).

[26] T. Bodineau and B. Derrida, J. Stat. Phys. 123, 277 (2006).

[27] T. Bodineau and B. Derrida, C. R. Physique 8, 540 (2007).

[28] B. Derrida, J. Stat. Mech. (2007) P07023.

[29] L. Bertini, A. De Sole, D. Gabrielli, G. Jona-Lasinio, and
C. Landim, Phys. Rev. Lett. 94, 030601 (2005).

[30] L. Bertini, A. De Sole, D. Gabrielli, G. Jona-Lasinio, and C. Landim, J. Stat. Phys. 123, 237 (2006).

[31] T. Bodineau and B. Derrida, Phys. Rev. E 72, 066110 (2005).

[32] C. Appert-Rolland, B. Derrida, V. Lecomte, and F. van Wijland, Phys. Rev. E 78, 021122 (2008).

[33] A. Imparato, V. Lecomte, and F. van Wijland, Phys. Rev. E 80, 011131 (2009).

[34] P. I. Hurtado, C. Pérez-Espigares, J. J. del Pozo, and P. L. Garrido, Proc. Natl. Acad. Sci. 108, 7704 (2011).

[35] T. Nemoto and S.-i. Sasa, Phys. Rev. E 83, 030105(R) (2011).

[36] C. W. Gardiner, Handbook of Stochastic Methods for Physics, Chemistry, and the Natural Sciences (SpringerVerlag, Berlin, 1983).

[37] P. Reimann, Phys. Rep. 361, 57 (2002).

[38] M. Doi and S. F. Edwards, The Theory of Polymer Dynamics (Clarendon Press, Oxford, 1986).

[39] P. Reimann, C. Van den Broeck, H. Linke, P. Hänggi, J. M. Rubi, and A. Pérez-Madrid, Phys. Rev. Lett. 87, 010602 (2001).

[40] P. Reimann, C. Van den Broeck, H. Linke, P. Hänggi, J. M. Rubi, and A. Pérez-Madrid, Phys. Rev. E 65, 031104 (2002).

[41] V. Blickle, T. Speck, C. Lutz, U. Seifert, and C. Bechinger, Phys. Rev. Lett. 98, 210601 (2007).

[42] J. R. Gomez-Solano, A. Petrosyan, S. Ciliberto, R. Chetrite, and K. Gawȩdzki, Phys. Rev. Lett. 103, 040601 (2009).

[43] S. Toyabe, T. Okamoto, T. Watanabe-Nakayama, H. Taketani, S. Kudo, and E. Muneyuki, Phys. Rev. Lett. 104, 198103 (2010).

[44] G. S. Agarwal, Z. Phys. 252, 25 (1972).

[45] H. Risken, The Fokker-Planck Equation (SpringerVerlag, Berlin, 1984).

[46] J. Mehl, T. Speck, and U. Seifert, Phys. Rev. E 78, 011123 (2008).

[47] S. Dorosz and M. Pleimling, Phys. Rev. E 83, 031107 (2011).

[48] T. Speck and U. Seifert, J. Phys. A 38, L581 (2005).

[49] A. Imparato and L. Peliti, Europhys. Lett. 70, 740 (2005).

[50] A. Imparato and L. Peliti, Phys. Rev. E 72, 046114 (2005).

[51] J. M. Burgers, The Nonlinear Diffusion Equation Asymptotic Solutions and Statistical Physics (Reidel, Dordrecht, 1974). 
[52] M. D. Donsker and S. R. Varadhan, Commun. Pure Appl. Math. 28, 1 (1975).

[53] C. Maes and K. Netočný, J. Math. Phys. 48, 053306 (2007).

[54] J. P. Garrahan, R. L. Jack, V. Lecomte, E. Pitard, K. van Duijvendijk, and F. van Wijland, J. Phys. A 42, 075007 (2009).

[55] M. Baiesi, C. Maes, and B. Wynants, J. Stat. Phys. 137, 1094 (2009).

[56] C. Maes, K. Netočný, and B. M. Shergelashvili, Phys. Rev. E 80, 011121 (2009).

[57] C. Maes, K. Netočný, and B. Wynants, Physica A 387, 2675 (2008).

[58] S. Ono, Adv. Chem. Phys. 3, 267 (1961).

[59] K. Hayashi and S.-i. Sasa, Phys. Rev. E 69, 066119 (2004).

[60] T. Harada and S.-i. Sasa, Phys. Rev. Lett. 95, 130602 (2005).

[61] T. Speck and U. Seifert, Europhys. Lett. 74, 391 (2006).

[62] R. Chetrite, G. Falkovich, and K. Gawedzki, J. Stat. Mech. (2008) P08005.
[63] J. Prost, J.-F. Joanny, and J. M. R. Parrondo, Phys. Rev. Lett. 103, 090601 (2009).

[64] M. Baiesi, C. Maes, and B. Wynants, Phys. Rev. Lett. 103, 010602 (2009).

[65] D. S. Dean, J. Phys. A 29, L613 (1996).

[66] T. Bodineau, B. Derrida, and J. L. Lebowitz, J. Stat. Phys. 131, 821 (2008).

[67] Y. Oono and M. Paniconi, Prog. Theor. Phys. Suppl. 130, 29 (1998).

[68] T. Hatano and S.-i. Sasa, Phys. Rev. Lett. 86, 3463 (2001).

[69] S.-i. Sasa and H. Tasaki, J. Stat. Phys. 125, 125 (2006).

[70] E. Seneta, Non-Negative Matrices and Markov Chains, 2nd ed. (Springer, New York, 2006).

[71] K. Sekimoto, J. Phys. Soc. Jpn. 66, 1234 (1997).

[72] K. Sekimoto, Stochastic Energetics (Lecture Notes in Physics) (Springer, Berlin, 2010).

[73] K. Tomita and H. Tomita, Prog. Theor. Phys. 51, 1731 (1974). 